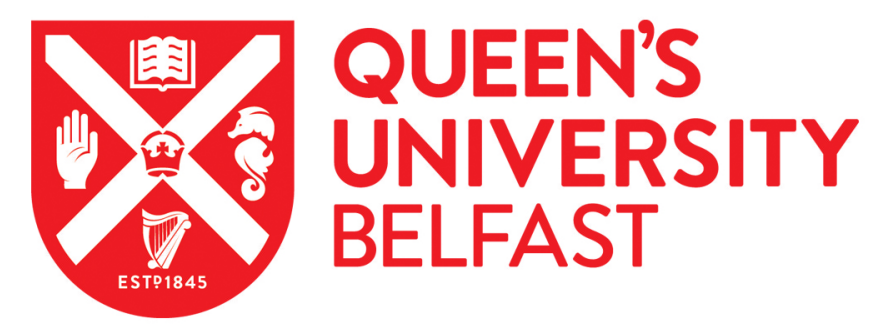

\title{
Influence of microstructure on the cutting behaviour of silicon
}

Goel, S., Kovalchenko, A., Stukowski, A., \& Cross, G. (2016). Influence of microstructure on the cutting behaviour of silicon. Acta Materialia, 105, 464-478. https://doi.org/10.1016/j.actamat.2015.11.046

\author{
Published in: \\ Acta Materialia
}

\section{Document Version:}

Peer reviewed version

Queen's University Belfast - Research Portal:

Link to publication record in Queen's University Belfast Research Portal

\section{Publisher rights}

(c) 2015 Crown Copyright. This manuscript version is made available under the CC-BY-NC-ND 4.0 license

http://creativecommons.org/licenses/by-nc-nd/4.0/ which permits distribution and reproduction for non-commercial purposes, provided the author and source are cited.

\section{General rights}

Copyright for the publications made accessible via the Queen's University Belfast Research Portal is retained by the author(s) and / or other copyright owners and it is a condition of accessing these publications that users recognise and abide by the legal requirements associated with these rights.

Take down policy

The Research Portal is Queen's institutional repository that provides access to Queen's research output. Every effort has been made to ensure that content in the Research Portal does not infringe any person's rights, or applicable UK laws. If you discover content in the Research Portal that you believe breaches copyright or violates any law, please contact openaccess@qub.ac.uk. 


\title{
Influence of microstructure on the cutting behaviour of silicon
}

\author{
Saurav Goel ${ }^{\mathrm{a} *}$, Andrii Kovalchenko ${ }^{\mathrm{b}}$, Alexander Stukowski ${ }^{\mathrm{c}}$ and Graham Cross ${ }^{\mathrm{d}, \mathrm{e}}$ \\ ${ }^{\text {a }}$ School of Mechanical and Aerospace Engineering, Queen's University, Belfast, BT95AH, UK \\ ${ }^{\mathrm{b}}$ Institute for Problems of Materials Science, National Academy of Sciences, Kyiv, 03142, Ukraine \\ ${ }^{\mathrm{c}}$ Institute of Materials Science, Darmstadt University of Technology, Darmstadt, D-64287, Germany \\ ${ }^{\mathrm{d}}$ CRANN Nanoscience Institute, School of Physics, Trinity College, Dublin 2, Ireland \\ ${ }^{\mathrm{e} A d a m a}$ Innovations Limited, Dublin 2, Ireland \\ * Corresponding author Tel.: +44 289097 5625, Email address: s.goel@qub.ac.uk, Fax: +44 02890974148
}

\begin{abstract}
:
We use molecular dynamics simulation to study the mechanisms of plasticity during cutting of monocrystalline and polycrystalline silicon. Three scenarios are considered: (i) cutting a single crystal silicon workpiece with a single crystal diamond tool, (ii) cutting a polysilicon workpiece with a single crystal diamond tool, and (iii) cutting a single crystal silicon workpiece with a polycrystalline diamond tool. A long-range analytical bond order potential is used in the simulations, providing a more accurate picture of the atomic-scale mechanisms of brittle fracture, ductile plasticity, and structural changes in silicon. The MD simulation results show a unique phenomenon of brittle cracking typically inclined at an angle of $45^{\circ}$ to $55^{\circ}$ to the cut surface, leading to the formation of periodic arrays of nanogrooves in monocrystalline silicon, which is a new insight into previously published results. Furthermore, during cutting, silicon is found to undergo solid-state directional amorphisation without prior Si-I to $\mathrm{Si}$-II (beta tin) transformation, which is in direct contrast to many previously published MD studies on this topic. Our simulations also predict that the propensity for amorphisation is significantly higher in single crystal silicon than in polysilicon, signifying that grain boundaries eases the material removal process.
\end{abstract}

Keywords: MD simulation; silicon; diamond; nanometric cutting; uniaxial tensile testing. 
Paper accepted in the Journal "Acta Materialia" in November 2015

Abbreviations:

$\begin{array}{ll}A B O P & \text { Analytical bond order potential } \\ B D T & \text { Brittle-ductile transition } \\ \text { CMOS } & \text { Complementary metal-oxide-semiconductor } \\ d & \text { Uncut chip thickness } \\ \text { DXA } & \text { Dislocation extraction algorithm } \\ F x & \text { Tangential cutting force } \\ F y & \text { Thrust force } \\ \text { GB } & \text { Grain boundary } \\ \text { HPPT } & \text { High pressure phase transformation } \\ M O S F E T & \text { Metal-oxide-semiconductor field-effect transistor } \\ \text { NVE } & \text { Microcanonical ensemble } \\ \text { OVITO } & \text { Open Visualization Tool } \\ \text { Pbc } & \text { Periodic boundary conditions } \\ \text { PC } & \text { Polycrystalline } \\ \text { SC } & \text { Single crystal } \\ \text { UNCD } & \text { Ultra nanocrystalline diamond } \\ & \end{array}$

\section{Introduction}

Silicon has been used extensively in both single crystal as well as in polycrystalline form for a wide range of microelectronic applications including solar cells and conducting gates for CMOS and MOSFET processing devices [1]. Recent technological trends have also led to the synthesis of nanospheres and nanowires of silicon, which would potentially provide an even broader range of applications. However, before this shapes into a reality, it is important to fully understand and characterize the mechanical response of silicon to be able to regulate its behaviour across several disparate engineering applications. Consequently, strenuous efforts are being made to continue 
Paper accepted in the Journal "Acta Materialia" in November 2015

Moore's Law (which says that the count of transistors on a silicon chip doubles every two years.)

This will require production of ultra-thin silicon wafers. Moore's Law is not a fundamental law of nature, and sustaining this extraordinary rate of progress requires advances in our understanding and the ability to control the properties of materials. One of these properties is the ductile-brittle transition which limits the production of silicon wafers. Therefore, exploration and understanding of the mechanical properties and failure mechanisms of silicon and other nominal brittle materials have become an interesting research topic. In this regard, the existing research details several aspects of silicon, but this material is so versatile that many new phenomena are still being explored to bridge the missing gaps in our existing understanding. Across a number of those research studies, ductility in silicon by large has been attributed either to the occurrence of high pressure phase transformation (HPPT) [1], crystal twinning [2] or surface nucleation of dislocations [3, 4]. It is understood that the nucleation of dislocations is more prevalent than HPPT in the presence of free surfaces, for examples in, nanoparticles of silicon [5] while no evidence of crystal twinning during contact loading of silicon has been reported in the literature other than the work of Mylvaganam et al. [2]. Reports of HPPT of silicon on the other hand have a richer history [6], and in the past, several phases of silicon were identified [7] by post-experimentation analysis, which are summarised in Table I along with the typical stress levels at which these phases persist.

Table I: Various high-pressure phases of silicon [7]

\begin{tabular}{|c|c|c|}
\hline Phase of Silicon & Lattice structure & Stress (GPa) \\
\hline Pristine Si-I (brittle) & Diamond cubic & 0 to 12.5 \\
\hline Si-II (metallic) & $\begin{array}{c}\text { Body centred tetragonal (Beta- } \\
\text { Sn) }\end{array}$ & 0 to 2.1 (ambient) \\
\hline Si-III or bc8 & Body centred cubic (BCC) & 14 to 35 \\
\hline Si-IV & $\begin{array}{c}\text { Hexagonal diamond } \\
\text { (Lonsdaleite) }\end{array}$ & Martensitic transformation from Si-I \\
\hline Si-V & Primitive Hexagonal & 40 to 78.3 \\
\hline Si-VI & Unidentified & 0 to 14.8 \\
\hline Si-VII & Hexagonal close packed & Tetragonal (30 atoms/unit cell) \\
\hline Si-VIII &
\end{tabular}


Paper accepted in the Journal "Acta Materialia" in November 2015

\begin{tabular}{|c|c|c|}
\hline Si-IX & Tetragonal (12 atoms/unit cell) & $\begin{array}{c}0 \text { to } 12 \\
\text { (Upon rapid decompression from Si-II }\end{array}$ \\
\hline Si-X & Face centred cubic (FCC) & 78.3 to 230 \\
\hline Si-XI (Imma) & Body centred orthorhombic & 13 to 15 \\
\hline Si-XII (R8) & Trigonal (8 atoms/unit cell) & 2 to 12 \\
\hline
\end{tabular}

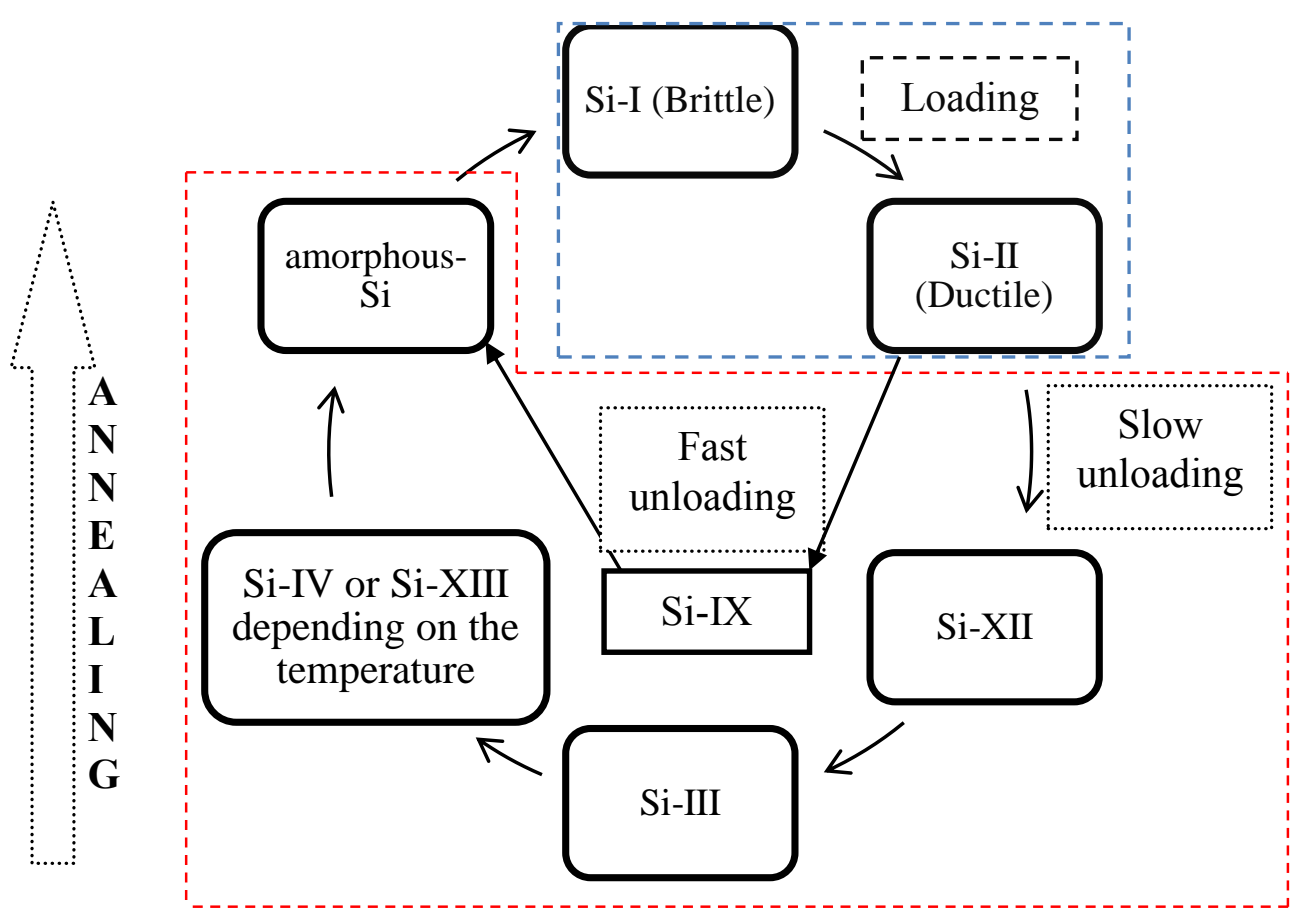

Figure 1: High pressure phase transformation of silicon during its contact loading [1] - Blue line signifies high pressure phase transformation of silicon to cause metallisation while the red line signifies back transformation after HPPT to an atmospheric phase depending on the rate of release of load.

A systematic representation of what happens to silicon during contact loading (nanoindentation, nanoimpact, loading in a diamond anvil cell and nanometric cutting) is shown in figure 1. Literature suggest that a critical magnitude of stress leads to metallization of silicon ( $\mathrm{Si}$-I transforms to metastable Si-II phase), and it is this process which facilitates a brittle-ductile transition $[8,9]$ in silicon during contact loading [10]. Thus, our current understanding is that HPPT facilitates a brittleductile transition, which in turn enables its ductile-regime machining as revealed in experiments [11]. Subsequently, the kinetics of structural transformation in silicon depends on the rate at which the pressure or load is released. As shown in figure 1, upon release of load, the metastable phase of Si-II transforms to amorphous silicon, although, different phases other than Si-I and Si-II appear depending 
Paper accepted in the Journal "Acta Materialia" in November 2015

on the release rate. The transformation from high pressure metallic phase (Si-II) to an atmospheric phase (a-Si) is usually accompanied by a volume expansion of $\sim 10 \%$, contributing to the elastic recovery of the cut surface.

Molecular dynamics (MD) simulation has played a key role in developing our understanding of such processes, and an overview of insights gained from atomic-scale modelling of diamond machining of silicon has recently been given by the authors [1]. Typically, three-body potentials of the Tersoff [12, 13] or the analytical bond order potential (ABOP) [14] type have been used in the past to model the interaction between $\mathrm{Si}$ and $\mathrm{C}$ atoms. These potentials are short ranged and yield ductile instead of brittle behavior for covalent materials such as silicon or diamond. This shortcoming of the potentials used in the past raises questions about previously performed MD studies of nanometric cutting processes. Pastewka et al. [15] recently developed an improved screened cutoff scheme, which extends the range of these potentials to overcome the described limitations. These new, screened potential formulations, which will be used in this simulation study, correctly describe the brittle materials response and improved description of amorphous phases.

In addition to the quality of the employed interatomic potential used in an MD simulation study, an analysis of the mechanism of deformation and a precise identification of phases are crucial. Following experimental reports, many atomistic simulation studies of nanoindentation and other surface nanomodification processes have described a Si-I to Si-II phase transition to occur at pressures starting at $12 \mathrm{GPa}$ [16-18]. However, the evidence presented by these simulation-based reports for the presence of the Si-II crystalline phase remains only suggestive. Typically, a change in the radial distribution function computed from the atomic positions and the appearance of some highly coordinated atoms are presented as indications that a phase transformation has taken place. However, it must be noted that these analysis tools cannot give conclusive proof of the presence of an ordered crystalline phase, especially if it comprises only a relatively small group of atoms. In particular, the direct amorphisation of the cubic diamond phase of silicon cannot be excluded, as it would manifest itself in a very similar way. A phase stability analysis based on classical potentials and ab-initio calculations [19] has shown 
Paper accepted in the Journal "Acta Materialia" in November 2015

that a homogeneous transition of cubic diamond (Si-I) to the beta-Sn ( $\mathrm{Si}-\mathrm{II})$ phase of silicon sets in at a critical pressure of $64 \mathrm{GPa}$, which is considerably higher than the reported average stress levels reached in contact loading simulations. Even though the Si-II phase becomes thermodynamically stable at pressures as low as $8 \mathrm{GPa}$, a kinetic activation barrier can delay the phase transition in a perfect and homogeneous crystal, which is the typical model situation in MD studies. Under these circumstances, doubts remain as to whether the atomic arrangements seen in MD simulations really represent a genuine Si-II crystalline phase.

Extant literature also details that, in addition to HPPT, there are several other processes that occur simultaneously during nanometric cutting of silicon. Examples are an elastic-plastic transition of the material, movement and propagation of dislocations and varying reactions of grain boundaries in the cutting zone [20], all of which are influenced by the crystallographic structure, orientation and microstructure of the material. In this context, polycrystalline silicon (polysilicon) is extensively used in the production of solar cells making use of monocrystalline or multi-crystalline silicon for substrates. These crystalline type silicon solar cells account for over $90 \%$ of the worldwide production of solar cells. In the literature, no attempt was made to clarify whether the deformation of single crystal silicon and polysilicon occurs in the same way or not. Similarly, ultra nanocrystalline diamond (UNCD) is gaining significant research interest across a range of engineering disciplines mainly because it rivals single crystal diamond in terms of high hardness [21] and low coefficient of friction. Comparing the machining performance of a UNCD cutting tool against a single crystal cutting tool is therefore a natural intellectual curiosity. In view of the aforementioned issues, we identified the following key research questions, which will be addressed in this paper:

1. Will the use of improved interatomic potentials confirm or contradict previous MD simulations, and shed more light on aspects of fracture, wear and plasticity of silicon that are known from experiment but were not previously observed in simulations based on other potentials?

2. What mechanism prevails during ductile-regime cutting of silicon: high pressure phase 
Paper accepted in the Journal "Acta Materialia" in November 2015

transformation, crystal twinning or nucleation of dislocations?

3. Will the occurrence and extent of high pressure phase transformation be the same in both single crystal and polysilicon during nanometric cutting?

4. How does the presence of shear such as in nanometric cutting influences the failure mode of silicon in contrast to hydrostatic compression such as in diamond anvil cell?

5. What are the implications of the microstructure on the machining stress and cutting forces?

Furthermore, a general argument against conventional atomistic simulations may be made that real world materials are never defect-free. The typical defects observed in real world materials are impurities, voids, dislocations, and grain boundaries. Machining of realistic materials (with defects), such as polycrystalline structures, in which the major defects are grain boundaries, is of great importance to real-world applications. Hitherto, no simulation investigation have been performed in this direction because of the complexities involved in constructing the polycrystalline model and the large system sizes and long computation times required. Considering these aspects, it is important to conduct an MD study using an appropriate potential function to identify the role of microstructure of the silicon workpiece in influencing its plasticity, a question at the forefront of nanotechnology and yet to be answered. We note that the typical grain size in a polysilicon substrate could be a few microns, but modelling such a size using MD simulation is still prohibitive using current computational capabilities. Therefore, this work should be viewed as a test bed study involving grain sizes that are suited to the scale of MD.

\subsection{MD simulation details}

\subsection{Simulation setup and potential function}

This work employed LAMMPS, the "Large-scale atomic/molecular massively parallel simulator" (24 February 2015 version) [22] to perform the MD simulations, and OVITO [23] was used to visualize and analyze the results. Furthermore, the automated "dislocation extraction algorithm" (DXA) [24, 25] for identification of dislocations and other crystal defects was used. The MD simulation model after initial equilibration is shown in figure 2. Atoms of the workpiece (silicon atoms) and that of the 
Paper accepted in the Journal "Acta Materialia" in November 2015

diamond cutting tool (carbon atoms) were divided into three distinct zones, namely boundary atoms, thermostatic atoms and Newton atoms. The atoms in the Newton region, which is directly affected by the tool-workpiece interaction, followed Newtonian dynamics (NVE dynamics), while atoms in a thin boundary layer were subjected to a thermostat to dissipate the heat generated in the cutting region, which, in experiment, is dissipated by air or lubricant. Atoms at the outermost boundary of the model were fixed to keep the workpiece in place, while boundary atoms of the cutting tool were rigidly moved at constant velocity. Provided a suitable potential energy function is employed, MD simulation is a powerful research tool to understand the tribology, plasticity and wear behaviour of a range of materials [26-29]. Accordingly, the newly proposed potential energy function (a screened version [15] of the Si-II (silicon) developed by Erhart et al. [14]) was used in this study. Prior to use, we performed several tests to ensure its robustness by reproducing the elastic constants and other relevant mechanical properties of both silicon and diamond. Some of these properties, like the equilibrium lattice parameter, Young's modulus on the three crystallographic planes, Voigt Poisson's ratio, bulk modulus, shear modulus and shear strength of silicon and diamond at $0 \mathrm{~K}$, are listed in Table II and compared with experiments for reference. In addition to this, a simulation involving tensile pulling of a silicon nanowire was performed to examine the fracture stress and strain to cause rupture in silicon. Based on these assessments, we concluded that the potential function is amenable to study the aforementioned research problem of cutting of silicon. 

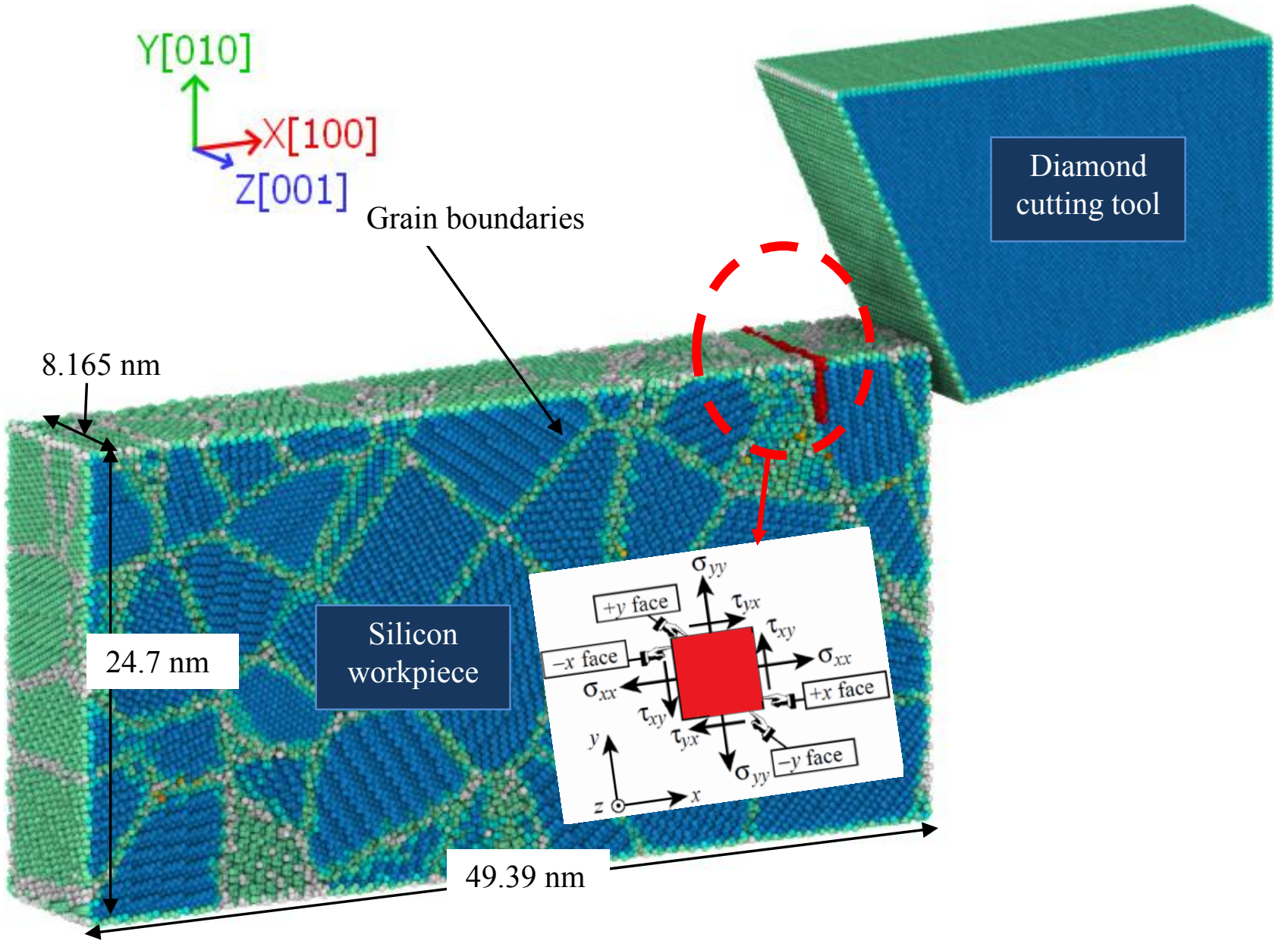

Figure 2: Schematic diagram of the MD simulation model of the nanometric cutting of polysilicon. A small volume of material (red group of atoms of dimension $0.7 \mathrm{~nm} \times 3 \mathrm{~nm} \times 8.165 \mathrm{~nm}$ ) was used to monitor the stress evolution in the cutting region. Blue atoms are crystalline diamond cubic atoms while green and white atoms form grain boundaries and free surfaces.

Table II: Material properties obtained from the screened bond order potential

\begin{tabular}{|c|c|c|c|c|}
\hline \multirow{2}{*}{ Properties $(\mathrm{T}=0 \mathrm{~K})$} & \multicolumn{2}{|c|}{ Silicon } & \multicolumn{2}{|c|}{ Diamond } \\
\hline & Simulation & $\begin{array}{c}\text { Experiment } \\
{[14]}\end{array}$ & Simulation & $\begin{array}{c}\text { Experiment } \\
{[14]}\end{array}$ \\
\hline Equilibrium lattice constant (Å) & 5.429 & 5.429 & 3.566 & 3.567 \\
\hline $\mathrm{C}_{11}=\mathrm{C}_{22}=\mathrm{C}_{33}(\mathrm{GPa})$ & 169 & 168 & 1088 & 1081 \\
\hline $\mathrm{C}_{12}=\mathrm{C}_{13}=\mathrm{C}_{23}(\mathrm{GPa})$ & 64.07 & 65 & 125.01 & 125 \\
\hline $\mathrm{C}_{44}=\mathrm{C}_{55}=\mathrm{C}_{66}(\mathrm{GPa})$ & 72 & 80 & 641 & 579 \\
\hline Young's modulus $\left(\mathrm{E}_{100}\right)[30](\mathrm{GPa})$ & 133.9 & 132 & 1062.6 & 1055 \\
\hline
\end{tabular}


Paper accepted in the Journal "Acta Materialia" in November 2015

\begin{tabular}{|c|c|c|c|c|}
\hline$C_{11}-2 \frac{C_{12}}{C_{11}+C_{12}} C_{12}$ & & & & \\
\hline $\begin{array}{l}\text { Young's modulus }\left(\mathrm{E}_{110}\right)[30](\mathrm{GPa}) \\
4 \frac{\left(C_{11}{ }^{2}+C_{12} C_{11}-2 C_{12}{ }^{2}\right) C_{44}}{2 C_{44} C_{11}+C_{11}{ }^{2}+C_{12} C_{11}-2 C_{12}{ }^{2}}\end{array}$ & 161.4 & 171 & 1231.6 & 1167 \\
\hline $\begin{array}{l}\text { Young's modulus }\left(\mathrm{E}_{111}\right)[30](\mathrm{GPa}) \\
3 \frac{C_{44}\left(C_{11}+2 C_{12}\right)}{C_{11}+2 C_{12}+C_{44}}\end{array}$ & 173.2 & 189 & 1300.6 & 1210 \\
\hline $\begin{array}{l}\text { Voigt Poisson's ratio [31] } \\
\frac{C_{11}+4 C_{12}-2 C_{44}}{4 C_{11}+6 C_{12}+2 C_{44}}\end{array}$ & 0.23 & 0.219 & 0.047 & 0.067 \\
\hline $\begin{array}{l}\text { Bulk Modulus(B) (GPa) [35] } \\
\frac{\left(C_{11}+C_{22}+C_{33}\right)+2\left(C_{12}+C_{13}+C_{23}\right)}{9}\end{array}$ & 99 & 99.3 & 446 & 443.7 \\
\hline $\begin{array}{l}\text { Shear modulus(G) }[34](\mathrm{GPa}) \\
\frac{C_{11}-C_{12}+C_{44}}{3}\end{array}$ & 58.9 & 61 & 534.9 & 512 \\
\hline Ideal shear strength $(\mathrm{G} / 2 \pi)(\mathrm{GPa})$ & 9.37 & 9.7 & 85.12 & 81 \\
\hline
\end{tabular}

A wealth of literature suggests $[32,33]$ that a very sharp tool should promote brittle-regime machining and for this reason, a sharp cutting edge was deliberately used in this study to probe brittle-regime dominated machining. The polycrystalline simulation models were generated by using an in-house developed Voronoi tessellation code [34, 35]. Further details of the MD simulation model are shown in Table III for the purpose of reproducibility.

Table III: Process variables and details of the MD simulation model

\begin{tabular}{|l|l|l|}
\hline Silicon workpiece dimensions & \multicolumn{1}{|l|}{$49.39 \mathrm{~nm} \times 24.7 \mathrm{~nm} \times 8.165 \mathrm{~nm}$, width of cut $(\mathrm{w})=$} \\
& $8.165 \mathrm{~nm}$ & In the diamond tool $=28$ \\
\hline Number of polycrystalline grains & In the silicon workpiece & Average grain size $=3 \mathrm{~nm}$ \\
& Average grain size $=5 \mathrm{~nm}$ & \\
\hline
\end{tabular}


Paper accepted in the Journal "Acta Materialia" in November 2015

\begin{tabular}{|c|c|}
\hline Uncut chip thickness $(d)$ & $3.08 \mathrm{~nm}=>(\mathrm{w} / \mathrm{d}$ ratio is 2.65$)$ \\
\hline $\begin{array}{l}\text { Workpiece cut surface and cutting } \\
\text { direction ( single crystal silicon) }\end{array}$ & $\left(\begin{array}{lll}0 & 1 & 0\end{array}\right)$ and $<100>$ \\
\hline $\mathrm{Si}-\mathrm{C}$ interatomic potential function & $\begin{array}{l}\text { Screening function [15] applied to Si-II (elemental } \\
\text { silicon) parameters of Erhart et al. [14] }\end{array}$ \\
\hline $\begin{array}{l}\text { Cutting tool (rake and clearance } \\
\text { angle) }\end{array}$ & $\begin{array}{l}\left(-25^{\circ} \text { and } 10^{\circ} \text { respectively) and extremely sharp edge }\right. \\
\text { (deformable) }\end{array}$ \\
\hline $\begin{array}{l}\text { Cutting distance (free travel + cutting } \\
\text { length) }\end{array}$ & $(1+29) \mathrm{nm}=30 \mathrm{~nm}$ \\
\hline Velocity of the tool & $250 \mathrm{~m} / \mathrm{s}=0.25 \mathrm{~nm} / \mathrm{ps}$ \\
\hline Total simulation time & $30 \mathrm{~nm} / 0.25 \mathrm{~nm} / \mathrm{ps}=120 \mathrm{ps}$ \\
\hline MD Timestep & $0.5 \mathrm{fs}=0.5 \times 10^{-3} \mathrm{ps}$ \\
\hline Total run timesteps & $120 / 0.0005=240,000$ \\
\hline
\end{tabular}

\subsection{Testing of the potential function using the uniaxial tensile test}

To augment support to our understanding of the nanometric cutting, preliminary tests were carried out to check the quality of the potential energy function through simulation of uniaxial tensile test of a silicon nanowire. The purpose of this test was purely to determine the fracture stress and strain of silicon at nanoscale under plane stress conditions and compare the results with other potential functions. Therefore, the uniaxial tests were carried out by using two other potential functions namely, a modified version of Tersoff's potential energy function [36] with applied screening, which was reported to predict the melting point of silicon correctly, and a potential function which was proposed to characterize the dislocation and defect behaviour in silicon appropriately, i.e. a spline-based Modified Embedded Atom Method (MEAM) potential energy function [37].

A silicon nanowire of $\phi 20.68 \mathrm{~nm}$ (cross sectional area $336 \mathrm{~nm}^{2}$ ) and length $48.98 \mathrm{~nm}$ was built (length/diameter ratio of 2.368) with the crystal orientation and direction of tensile pulling (parallel to the $\mathrm{Y}$ direction) as $<010>$. Periodic boundary conditions were applied in the Y direction and non- 
Paper accepted in the Journal "Acta Materialia" in November 2015

periodic and shrink-wrapped conditions in the $\mathrm{X}$ and $\mathrm{Z}$ directions. The wire was allowed to follow Newtonian dynamics (LAMMPS NVE dynamics) and was initially equilibrated at 300K with a time step of 0.5 fs. LAMMPS offer a command called "Fix deform" which may readily be used for straincontrolled tensile test simulations. The way this approach works is that the box length changes as per the function $\mathrm{L}(\mathrm{t})=\mathrm{L} 0(1+$ erate $\times \mathrm{dt})$ where erate is the applied engineering strain rate $\left(0.0005 / \mathrm{ps}=\left(5 \times 10^{8}\right) / \mathrm{sec}\right)$ and $\mathrm{dt}$ is the time elapsed. Plastic deformation in MD simulation at its limiting length and time scales is typically observed due to dislocation glide. Dislocation glide (in contrast to dislocation climb) is always associated with local shearing of atoms, which is why local shear strain is a useful measure to detect such activity. Therefore, to quantify the plastic deformation of the atoms, a measure proposed by Shimizu et al. [38] in the form of atomic local shear strain was chosen. A sample snapshot from the simulation result of silicon nanowire (at it its ultimate tensile strength) is shown in figure 3. The left part shows the typical atomic-shear strain to signify the appearance of a surface fissure which was observed to be the point of initiation of failure of the brittle silicon nanowire.

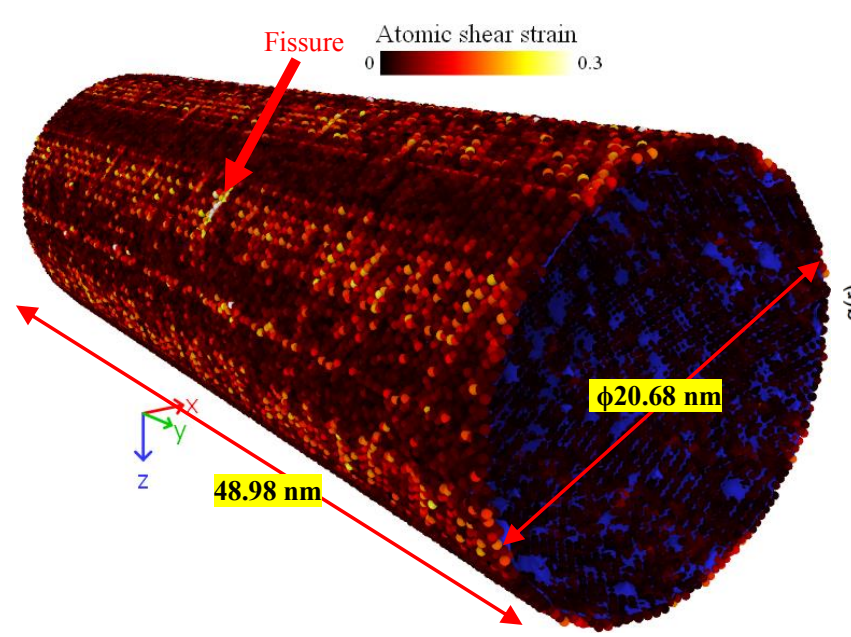

(a)

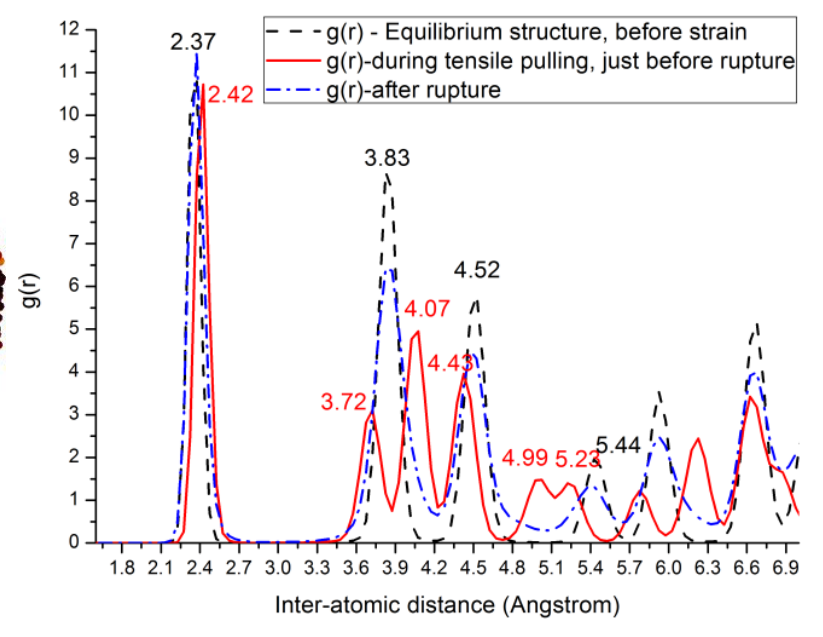

(b)

Figure 3: (a) Failure of silicon nanowire under uniaxial tensile pulling (left view shows the appearance of a surface fissure with the core atoms removed for ease of visualisation of changes in the microstructure of silicon. In the snapshot, blue crystallites are highly strained (metastable) clusters of silicon atoms and (b) the radial distribution function of the silicon nanowire at equilibrium stages just before rupture and after complete rupture

Just before rupture, the crystal structure of silicon inside the nanowire and on the surface of the nanowire was observed to be noticeably different from pristine crystalline material. This can also be 
Paper accepted in the Journal "Acta Materialia" in November 2015

seen from the radial distribution functions shown in figure $3 \mathrm{~b}$. In the equilibrium state (no strain applied), the radial distribution functions of the silicon nanowire showed four distinct peaks at interatomic distances of $2.37 \AA, 3.83 \AA, 4.52 \AA$ and $5.44 \AA$ (equilibrium lattice parameter) respectively, signifying perfectly four-fold symmetry of the diamond cubic silicon lattice. This configuration was observed to change as a result of significant elastic strain, leading to the formation of a different set of peaks at $2.42 \AA, 3.72 \AA, 4.07 \AA, 4.43 \AA, 4.99 \AA$ and $5.23 \AA$ just before rupture. After rupture, the elastically strained silicon lattice eventually reverted back to the original configuration having four distinct peaks (four fold coordination) with some remnants of amorphous silicon. Thus, it may be seen that the changes in the microstructure during loading are responsible for elastic-plastic transition in silicon. Furthermore, during the tensile test, no dislocations were observed for any of the tested potentials, at least for the applied strain rate of $\left(5 \times 10^{8}\right) /$ sec. Interestingly, a dummy trial performed at a higher strain rate of $\left(5 \times 10^{10}\right) / \mathrm{sec}$ showed some partial dislocation lines of $1 / 4<111>$ type Burgers vector family at several locations in the nanowire. A higher strain rate was also found to be accompanied by an increased rupture strain in all three potential cases by as much as $20 \%$. Thus, applied strain rate seems to make major influence on the dislocation mechanics of the silicon nanowire during its tensile pulling. In conjunction with figure 3, a comparison of the stress-strain curves obtained from the respective potential energy functions for the uniaxial test on silicon nanowire is shown in figure 4 . For the sake of information, the peak average temperature in the nanowire during rupture was obtained as $795 \mathrm{~K}, 670 \mathrm{~K}$ and $620 \mathrm{~K}$ respectively from the screened Erhart and Albe and modified Tersoff potentials and the MEAM potential respectively. 


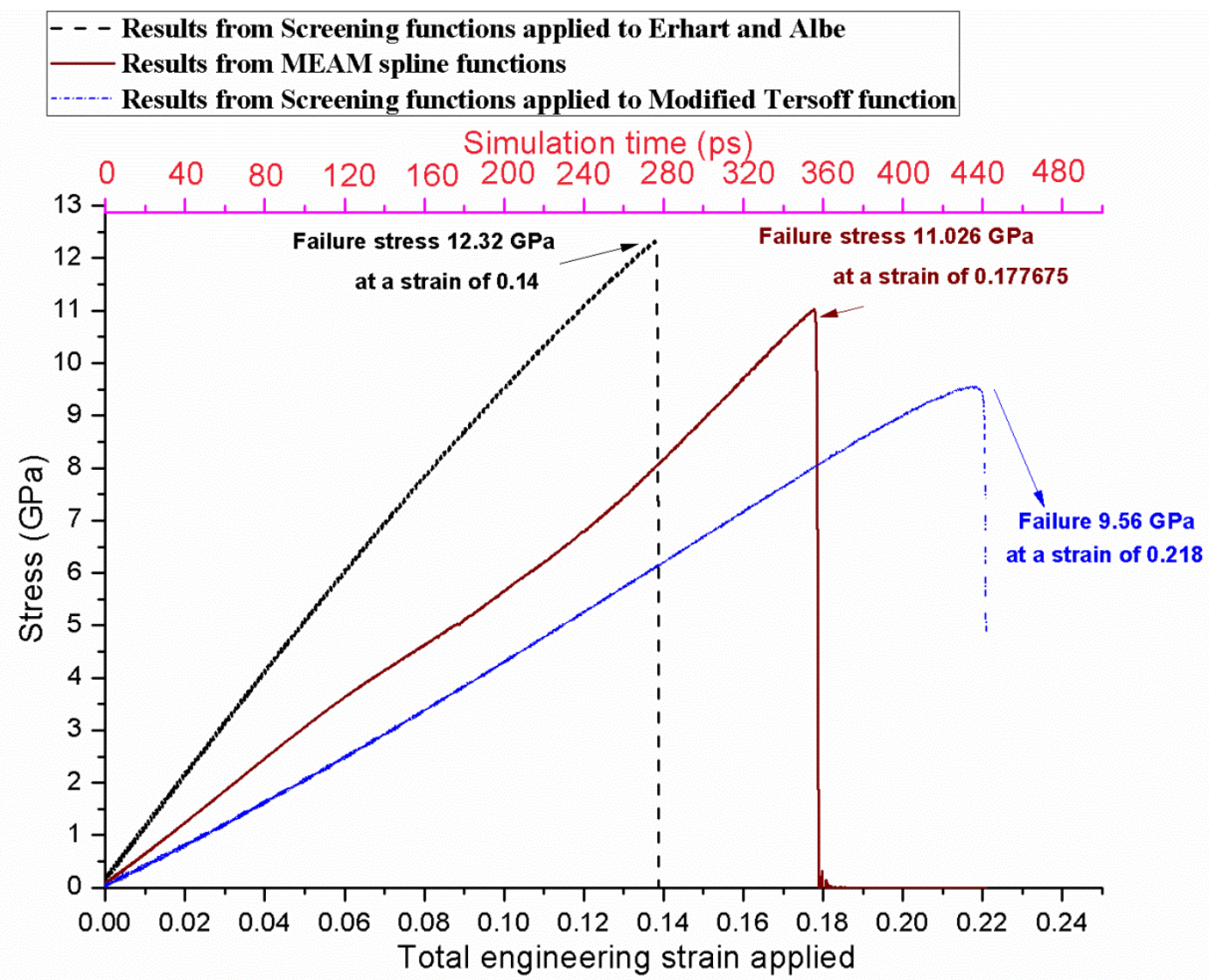

Figure 4: A typical comparison of the stress-strain curves obtained from the tensile test performed on a cylindrical nanowire of silicon having its initial temperature as $300 \mathrm{~K}$. A slight variation in the failure stress and failure strain predicted by different potential functions is evident.

As evident from figure 4, the three potential functions used in the study revealed three distinct values (slopes) of the elastic modulus (different fracture stress and fracture strains) i.e. $88 \mathrm{GPa}$ from screened Erhart and Albe potential, $62 \mathrm{GPa}$ from the MEAM potential and $43.85 \mathrm{GPa}$ from the screened Tersoff potential. Since the values of rupture stress and rupture strain predicted by the three potentials varies significantly, i.e., the value predicted by one potential is $50 \%$ compared to the value predicted by the other potential, this leaves us in a situation to question as to which potential function is more reliable. From a close survey of the literature [39], a value of around $60 \mathrm{GPa}$ to $80 \mathrm{GPa}$ was found to be the likely value of elastic modulus of a silicon nanowire of diameter $20 \mathrm{~nm}$, suggesting that the predictions of the Modified Tersoff function are less meaningful. Hence, the two obvious choices as a potential energy function for the simulation in this study for nanometric cutting are the MEAM spline potential function and the Erhart and Albe ABOP potential with applied screening function. One of the main advantages of the ABOP potential over the MEAM potential for this kind of study is 
Paper accepted in the Journal "Acta Materialia" in November 2015

that it readily offers the interaction potential between silicon and carbon and hence it is the obvious choice for performing the cutting simulations.

\section{Results and discussions of the nanometric cutting simulations}

\subsection{Cutting chips, Machining stresses and Cutting forces}

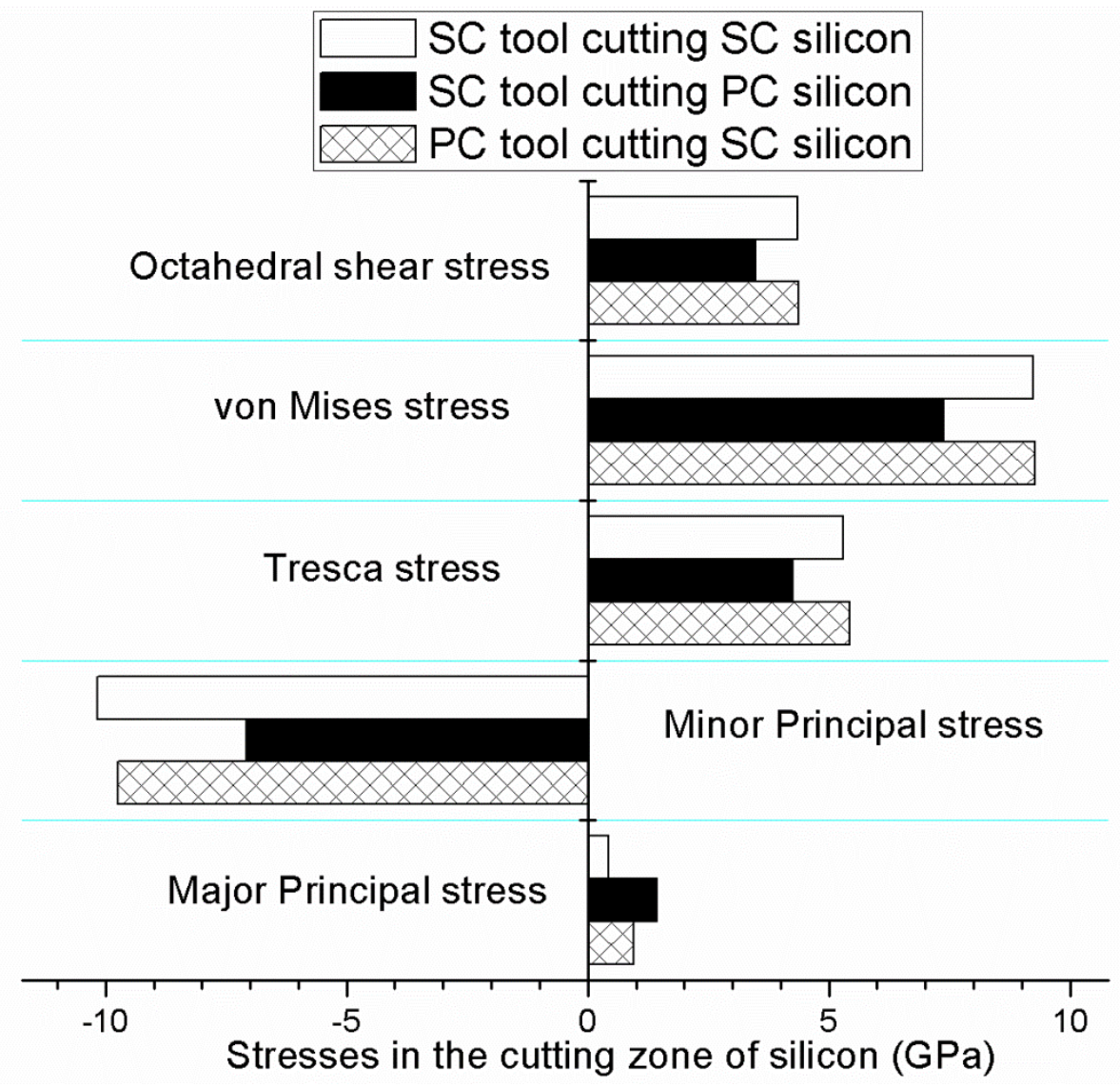

Figure 5: Peak average stress measures in the cutting region of the workpiece in three simulation test cases

It is believed that the von Mises and Tresca stress measures are more suited to predict yield in ductile materials whereas the Principal stress criterion is more suited to predict yielding in brittle materials [40]. Since it is not clear whether the deformation of silicon at the nanoscale is brittle or ductile, it makes sense to quantify the limiting stress for plastic flow of silicon using all stress measures. Accordingly, figure 5 shows the peak value of different stress measures in the monitored cutting region (highlighted in red colour in figure 2). The scalar stress values were determined by converting the atomic stress tensor to the physical stress tensor which was then fed to the formulas shown in Appendix-I to calculate Major Principal stress, Minor Principal stress, Tresca stress, von Mises stress 
Paper accepted in the Journal "Acta Materialia" in November 2015

and Octahedral shear stress. Independent of the stress measure used, the peak magnitude of stress while single crystal (SC) silicon was cut with a single crystal (SC) diamond tool is seemingly intermediate, while polycrystalline (PC) silicon specimen machined with a single crystal (SC) cutting tool showed the lowest magnitude.

It may be recalled that a diamond tool used in experiments is not atomically sharp, i.e., the edge radius is typically in few nanometres, which causes high compression in the cutting zone rather than high shear stress. In contrast to this, the cutting edge in the MD simulations is very sharp and hence Tresca stress is high. A multiscale simulation involving an experimental shape of the diamond tool will be needed to assert the exact magnitude of Tresca stress occurring in the experiments and the influence of shear leading to brittle-ductile transition (this will be expanded in future studies). In general, the von Mises and Tresca stress measures showed that cutting a single crystal silicon specimen with a polycrystalline (PC) diamond cutting tool requires the largest magnitude of stresses in the cutting zone. To confirm this observation, several simulations were performed on a single crystal silicon substrate at different cutting speeds from $250 \mathrm{~m} / \mathrm{s}$ down to $20 \mathrm{~m} / \mathrm{s}$. The peak magnitude of stress was the same in all cases, but the temperature in the cutting zone decreased with decreasing cutting speed. A snapshot of the local Minor Principal stress is shown in figure 6 to contrast the degree of compressive stress in the cutting zone in single crystal and polycrystalline silicon as well as at the grain boundaries in the PC substrate. The curliness in the cutting chip (especially in single crystal silicon) is emotive of the fact that the material removal process occurring during the simulation is in a ductile-regime, and hence occurs by the virtue of plastic deformation rather than fracture. This means that the Minor Principal (compressive) stress of about 12 GPa causes the silicon to exhibit plastic flow during its nanometric cutting and this is in line with the literature. 
Paper accepted in the Journal "Acta Materialia" in November 2015

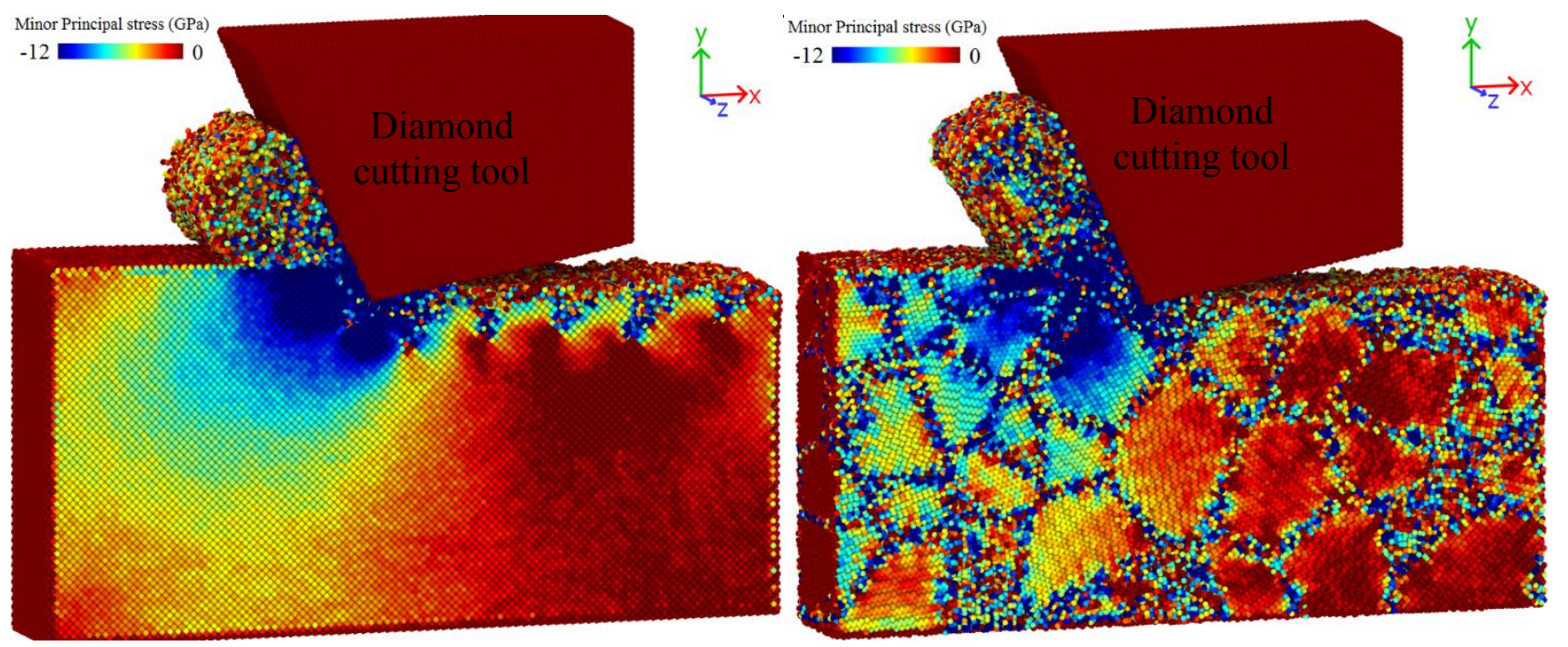

Figure 6: Variation in the Minor Principal stress (compressive) during cutting of (a) single crystal and (b) polycrystalline silicon workpiece

Figure 6 reveals that the chip morphologies of single crystal and polycrystal silicon are different. For example single crystal silicon chips show far more curl than the cutting chips of the polycrystalline silicon specimen. It is therefore important to find out the reasons underlying the differences between formation of cutting chips in SC and PC silicon specimens. To this end, figure 7 shows snapshots from the cutting simulation highlighting chip formation process in a PC substrate. We have used the structure analysis method implemented in OVITO, which can identify local atomic arrangements with cubic and hexagonal diamond structure. 


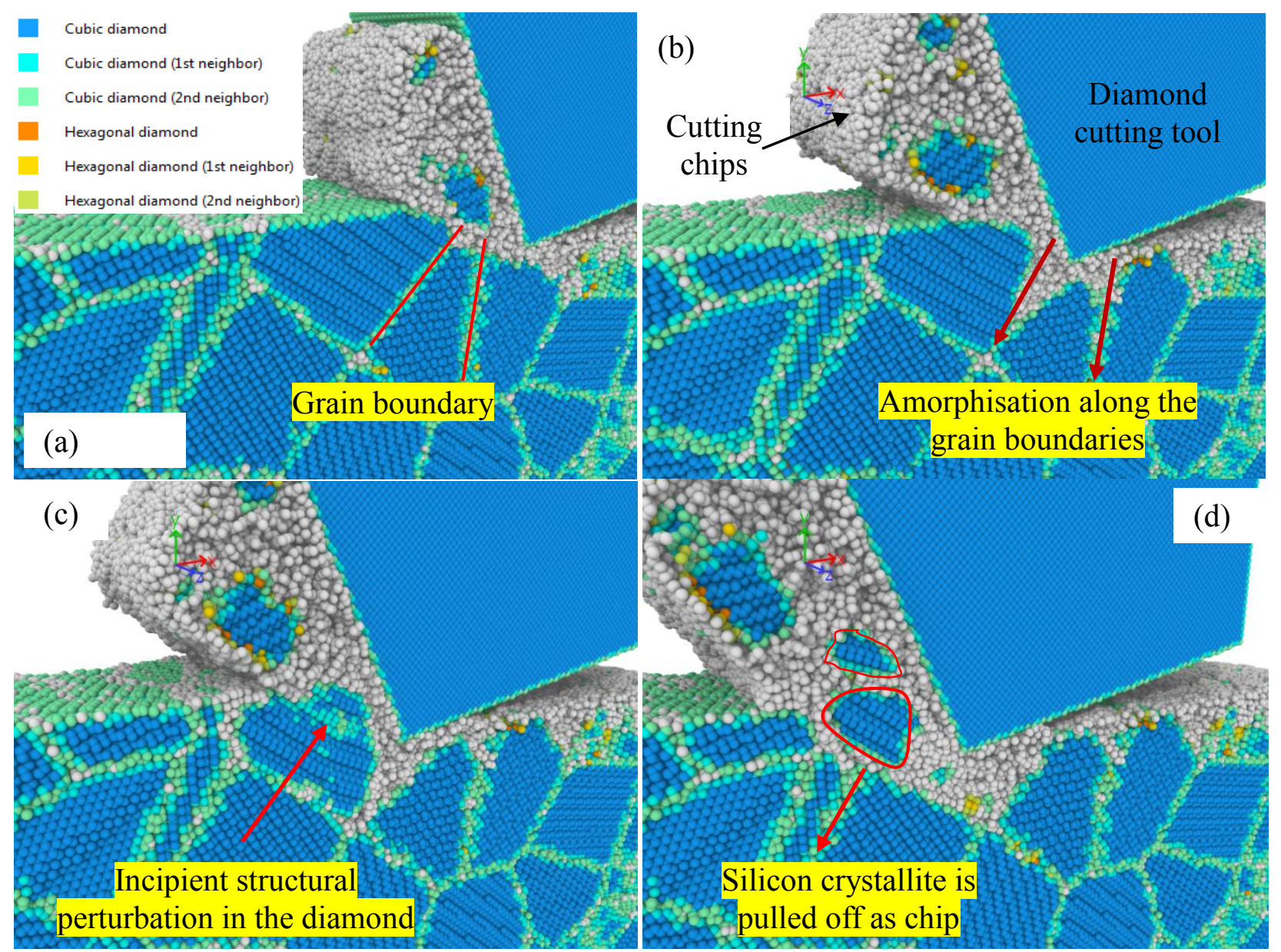

Figure 7: Snapshots from the MD simulation at various stages showing the process of material removal mechanism in a polycrystalline substrate and activity of amorphisation of silicon along the grain boundary and bisection of a grain highlighted in figure 7(c) and figure 7(d). Atoms are coloured here as per the module of Identify diamond structure scheme ${ }^{1}$

An interesting feature noted from figure 7 is how the material removal takes place in the PC specimen, i.e., the plastic deformation in silicon takes place preferentially along the grain boundaries rather than across the grain boundaries (figure 7b). As shown in figure 7c, when a grain lies ahead of the cutting tool, a portion of it undergoes amorphisation which is noticeable by the appearance of regions with disordered local structure. As the cutting tool advances further into the substrate, the grain may undergo partial transition depending upon the availability of slip systems and this facilitates splitting of the grain into two smaller grains. These clusters are composites of crystalline and amorphous phases of the pristine silicon that eventually emerge as the cutting chips. Furthermore, the grain boundaries shown earlier in figure $6 \mathrm{~b}$ exhibit large residual stresses, which aids in lowering the critical

\footnotetext{
${ }^{1}$ http://www.ovito.org/manual/particles.modifiers.identify_diamond_structure.html
} 
stress to activate plastic deformation compared to a single crystal silicon specimen. This suggests that grain boundaries assist in lowering the critical stress to cause plastic flow of a polycrystal compared to a perfect homogenous crystal. Consequently, it can be asserted that cutting of polycrystalline silicon is influenced by the movement of grains over each other in tandem with the structural transformation of silicon. We have verified and quantified this observation also from the measurement of the forces experienced by the cutting tool during the three simulations.
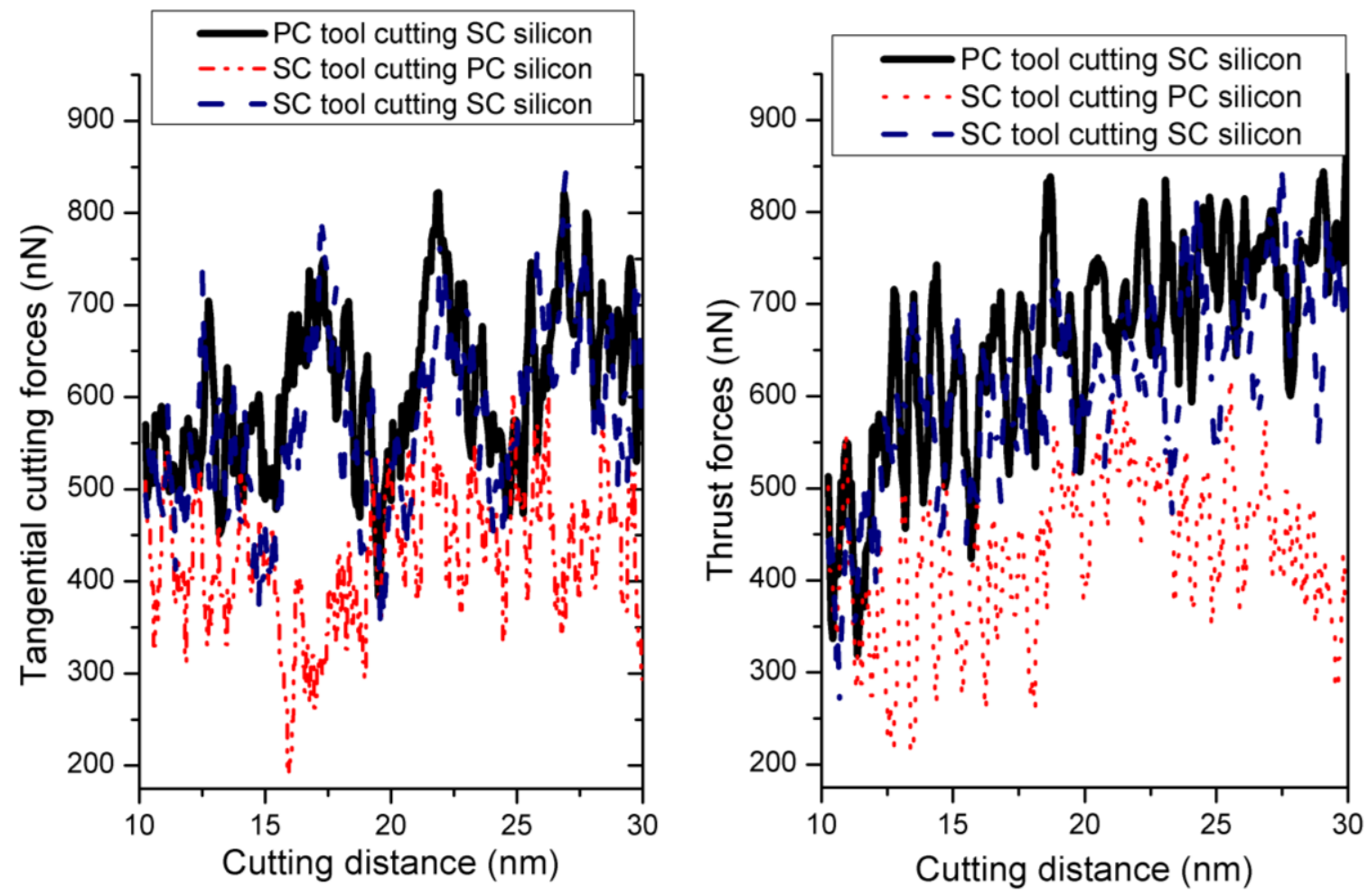

Figure 8: Steady state cutting forces in all three simulation test cases

Figure 8 shows the evolution of friction force (tangential cutting force) along the $x$ direction and normal force (thrust force) along the $y$ direction in all the three simulation test cases. The large noise in the cutting force signal reflects the discontinuous nature of wear, including plastic flow and formation and displacement of the cutting chips. It is particularly clear from the comparison of the cutting forces that the force acting on the cutting tool to machine a polycrystalline substrate was lowest while it was intermediate when using a single crystal tool to cut a single crystal substrate and maximum when a polycrystalline tool was used to cut a single crystal substrate. 


\subsection{Surface generation process and brittle fracture}

In this section we report a novel observation during the surface machining process. In contrast to any of the previously published simulation studies, our simulations showed a periodic occurrence of brittle cracking identifiable from the appearance of equidistant nanogrooves on the machined surface (figure 9a). In experiments, such nanogrooves have been observed in the SEM images of diamond turned silicon specimens (figure 9b), but the physics underlying their appearance was not highlighted [41]. A characteristic quality of these nanogrooves in both simulation and in the experiments (on the (010) orientation) is that they are oriented at an angle of between $45^{\circ}$ and $55^{\circ}$ to the direction of cutting. The physical origin of this observation can be seen from the fact that the cleavage face of the cut silicon substrate was oriented at an angle $45^{\circ}$ to the machined surface and that the stress on the tip of the nanogrooves shown earlier in figure 6a was hydrostatic compressive. We performed further characterization of these cracks as shown in figure 9 to gain more insights. Each atomic lattice layer of silicon (with $0.54 \mathrm{~nm}$ spacing) in figure 9 is coloured so as to visualize and quantify the depth of deformation. From figure 9, can be seen that the material in the cutting zone underneath the cutting tool undergoes shear as well as compression. The point on the cutting tool where sheared material separates from the compressed material is termed a point of stagnation. The layer of material above the point of stagnation (4th atomic layer coloured by black) leaves the workpiece in the form of cutting chips, while the layer of atoms underneath this black layer undergoes only compression (i.e. is not sheared) and therefore burnishes with the bottom (flank) face of the cutting tool. Consequently, at the point of stagnation there emerges a site of vorticity of the flowing silicon highlighted by the path $1 \rightarrow 2 \rightarrow 3$ in figure $9(\mathrm{c})$. The authors noticed that the phenomena of vorticity in plastically flowing solids in silicon [42] is common in other materials as well [43]. Energetically, it is favourable for the plastic phase of silicon to flow as cutting chips, but the portion of the material indicated by the black coloured layer of atoms is lying at such a depth that it neither shears nor compresses, and instead skids underneath the tool and flows downwards under the wake of the cutting edge. This region continuously burnishes with the tool and consequently, when the cutting tool moves past, the 
Paper accepted in the Journal "Acta Materialia" in November 2015

cutting edge becomes a point of vorticity.
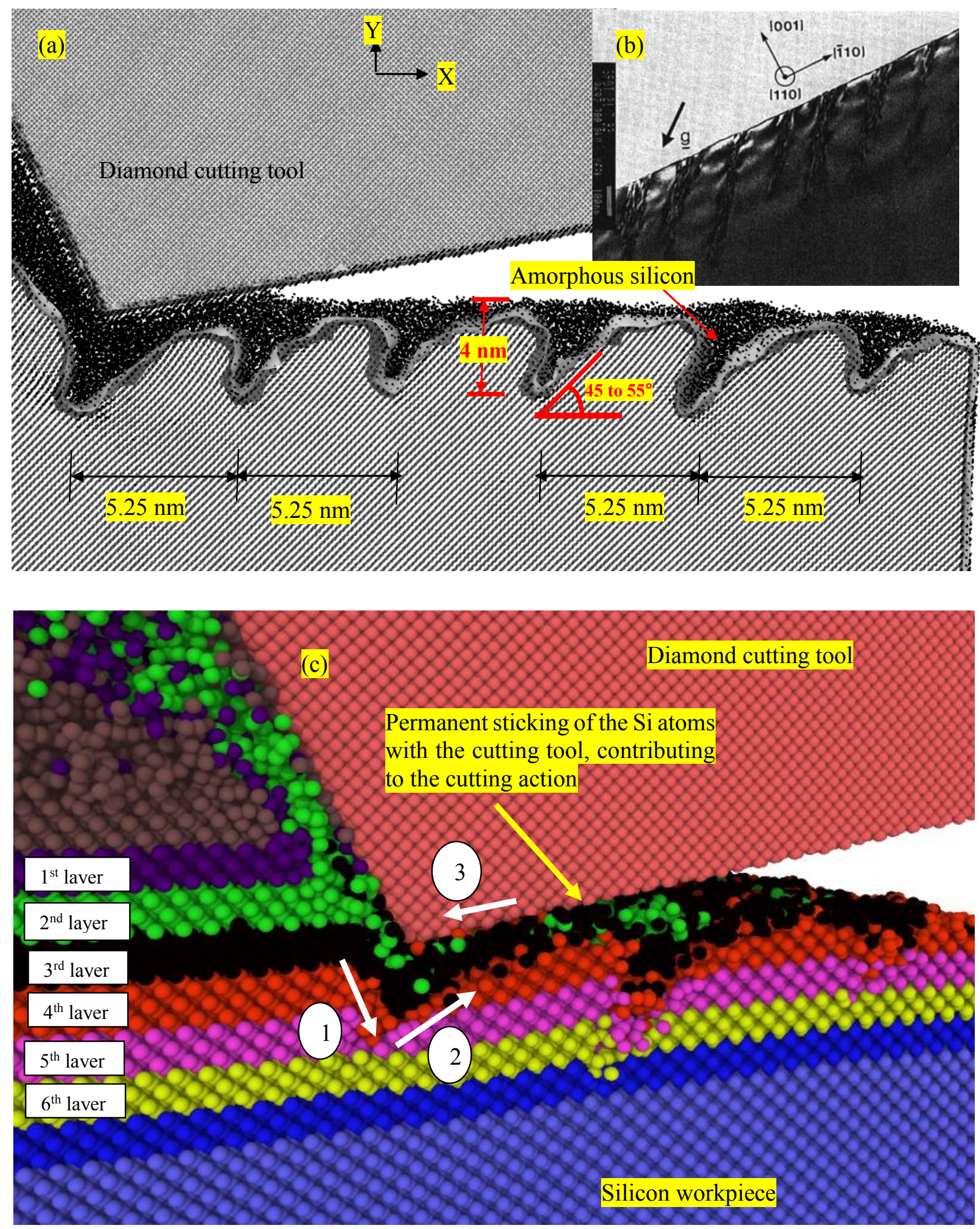

Figure 9: (a) Formation of nanogrooves in the cutting zone as a result of a layer of material that does not get sheared but instead burnishes with the cutting tool (b) Experimental image obtained from the diamond turned specimen on the (010) orientation showing periodic array of sub-surface damages

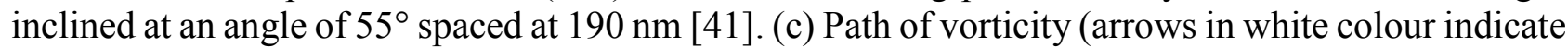
the movement of black coloured atoms akin to a fluid flow. 
Paper accepted in the Journal "Acta Materialia" in November 2015

The vorticity sites made by the moving tool are rich in amorphous remnants of silicon as all the excessive sheared chips (accumulating at regular intervals) are pushed to the sites where nanogrooves are formed. This cycle continues to repeat, periodic patterns keep forming, and thus brittle cracks are pressed and advances further by the pushing of the amorphous silicon. As noted by Lai et al. [44], the stagnation point for a particular tool varies only with the cutting depth, and is independent of the radius and critical angles of the tool. The implication of this phenomenon is that a more negative inclined tool edge (rake angle) will result in deeper sub-surface damage caused by the formation of such periodically appearing cavities. Notably, the authors have tested several other potential functions under similar conditions $[45,46]$, and it was found that such periodical grooves, which are known from cutting experiments, occur only with the screened empirical bond-order potential. This is a clear indication that the long-ranged screening function applied to the potential helps reproduce the brittle formation of surface cracks correctly.

\subsection{Structural transformations and mechanism of ductility in silicon}

High pressure phase transformations (HPPT) of brittle materials under high surface pressures is of particular interest in the field of contact loading because of the possibility of obtaining a ductile response from brittle materials. MD simulation has long been employed to understand such phenomenon, however, one of the main problems of empirical potentials is that they are optimized for a particular local environment of atomic configurations and were not originally designed to be employed in situations where the number of interacting neighbours changes abruptly, e.g. during contact loading where the second neighbours come within the range of what was defined to be the first-neighbour range [19]. In addition to deficient potentials, insufficient analysis techniques have been used to study HPPT in MD simulations. Specifically in silicon, the coordination number has long been used as the only local criterion to detect the formation of Si-II. It may however be noted that though the coordination number is a useful and necessary criterion, it is not a sufficient criterion to assert formation of Si-II in a region of the material (for example, a certain fraction of 6-fold coordinated atoms or atoms with higher coordination number can also be found in the amorphous 
phase) [16]. A structural analysis of the local atomic arrangement is required to unambiguously prove the existence of Si-II, an important step that has been skipped in previous simulation studies, possibly resulting in premature conclusions of $\mathrm{Si}-\mathrm{I}$ to $\mathrm{Si}$-II transformation being observed in the MD simulations

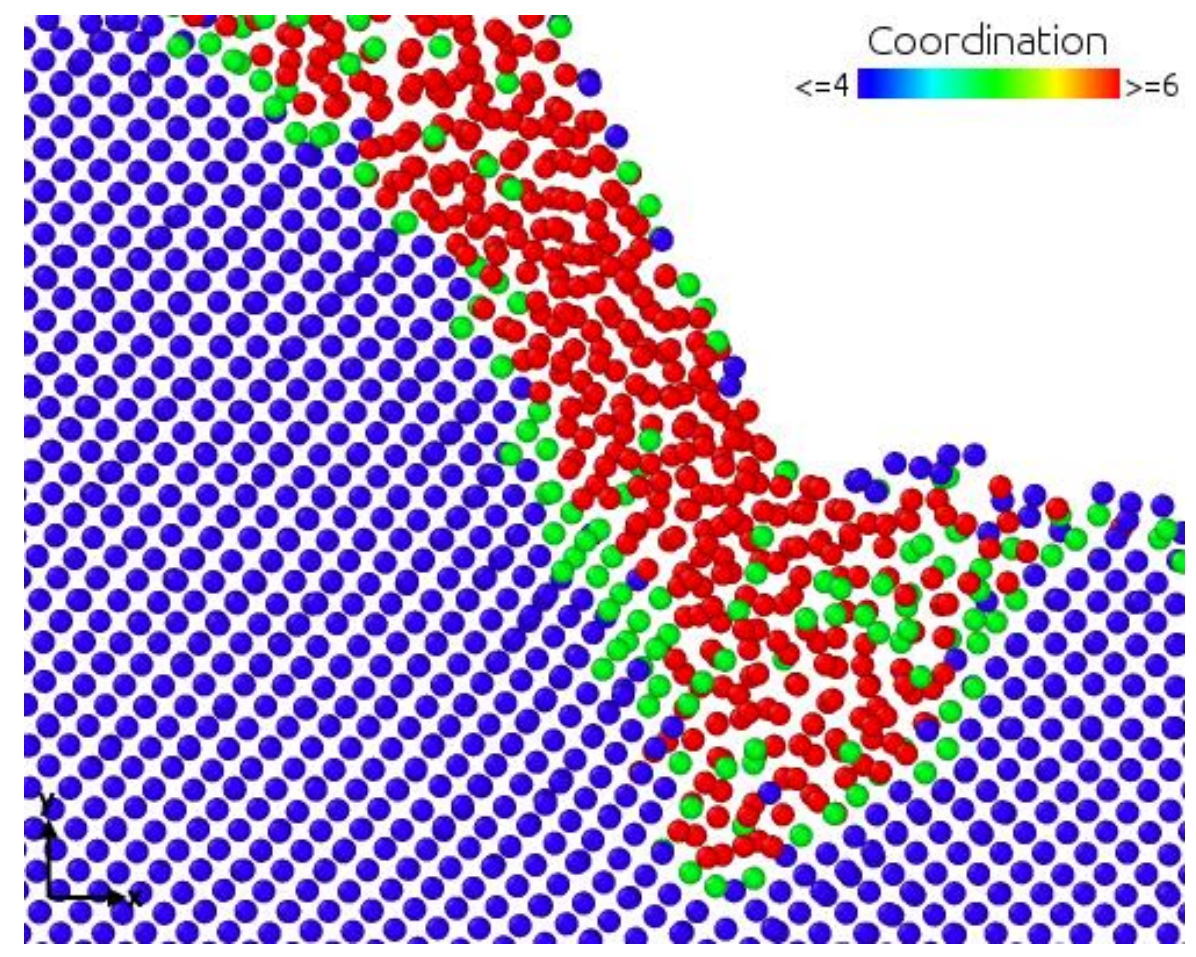

Figure 10: Atomic coordination numbers in the cutting zone of SC silicon (tool not shown).

It is therefore not surprising to learn that despite use of measures such as the coordination number and radial distribution function, there has been no distinct direct evidence presented in the literature concerning Si-I to Si-II of silicon during its simulated contact loading. Figure 10 shows the changes in the coordination number in the cutting zone of silicon. This may be seen most usefully in conjunction with simulation videos (provided as supplementary information to this paper). These both show that the cubic silicon crystal directly becomes amorphous in a short time scale of a few picoseconds under the influence of the cutting stresses. No crystalline phase of silicon other than SiI was observed in the cutting zone even in the simulation performed at a lower cutting speed (in a dummy trial). The wealth of literature suggests that the coordination number of 6 represents the formation of $\beta$-Sn phase ( $\mathrm{Si}$-II) silicon typically at a pressure of about $12 \mathrm{GPa}$ which is what has been 
Paper accepted in the Journal "Acta Materialia" in November 2015

verified experimentally as well as in the simulation performed in this work. However, in this regard, firstly, Gilman [47] asserts that the value of coordination number of $\beta$-Sn silicon cannot be perfectly 6 because there is always a difference of $5.6 \%$ between the subsequent nearest neighbour atoms and secondly, Mizushima et al. [19] used stability analysis and direct MD simulation to show that a pressure considerably higher than that predicted by the thermodynamic criterion is required to induce the transition from diamond-cubic to $\beta$-Sn structure in defect-free crystals such as the one used in simulations. They presented ab-initio calculations and classical MD simulation results using the Tersoff potential to demonstrate that the activation energy at the critical pressure needed to transform diamond cubic silicon to $\beta$-Sn structure $\mathrm{Si}$ is $0.3 \mathrm{eV} /$ atom, which is achieved typically at a pressure of about $64 \mathrm{GPa}$ at $300 \mathrm{~K}$. The process of Si-I to Si-II transformation is known to be an outcome of tetragonal shear instability of the silicon lattice structure. The materials used in experiment carry various types of lattice defects, and these defects lower the magnitude of the stress ( $\sim 12 \mathrm{GPa})$ required to cause $\mathrm{Si}-\mathrm{I}$ to $\mathrm{Si}-\mathrm{II}$ transformation. Another view of looking at the process of nanometric cutting in contrast to nanoindentation and hydrostatic compression is that it is a shear dominated process as opposed to compression dominated. Consequently, the presence of deviatoric stress conditions at the atomic scale results in combined bond stretching and distortion. This bond configuration state does not guarantee a state of tetragonal shear and hence direct amorphisation is more likely during the MD simulations rather than Si-I to Si-II transformation.

We have generated additional evidence for our observations by simulating X-Ray diffraction (XRD) and selected area electron diffraction intensity (SAED) patterns through use of the compute XRD and compute SAED features [48] of LAMMPS. In this algorithm, the c value which is a parameter to adjust the spacing of the reciprocal lattice nodes in the $h, k$ and 1 directions respectively is very important as this varies for individual materials. Prior to simulating XRD of silicon, we benchmarked silicon and the c value was found as 0.03 . To perform XRD and SAED of our cutting simulations, we made use of the same group of atoms which were monitored for stress in figure 2. In accord with this, we have shown the simulated XRD and SAED in figure 11 and figure 12 respectively. A close 
Paper accepted in the Journal "Acta Materialia" in November 2015

examination of the XRD spectra before cutting shows distinct peaks at $27.775^{\circ}, 46.225^{\circ}$ and $54.775^{\circ}$ corresponding to the standard XRD peaks in crystalline silicon known from silicon nanopowder experimental data [49]. During cutting, several scattered peaks were observed to appear which are emotive of the presence of amorphous silicon. The potential for the existence of a primitive hexagonal phase of silicon was raised by the emergence of small peaks at $33^{\circ}$ and $35^{\circ}[50]$, but this was rejected based on the fact that at lower angles no other additional strong peaks were observed. In accord with this, the SAED patterns shown in figure 12 also supported the observation of solid state amorphisation of silicon.

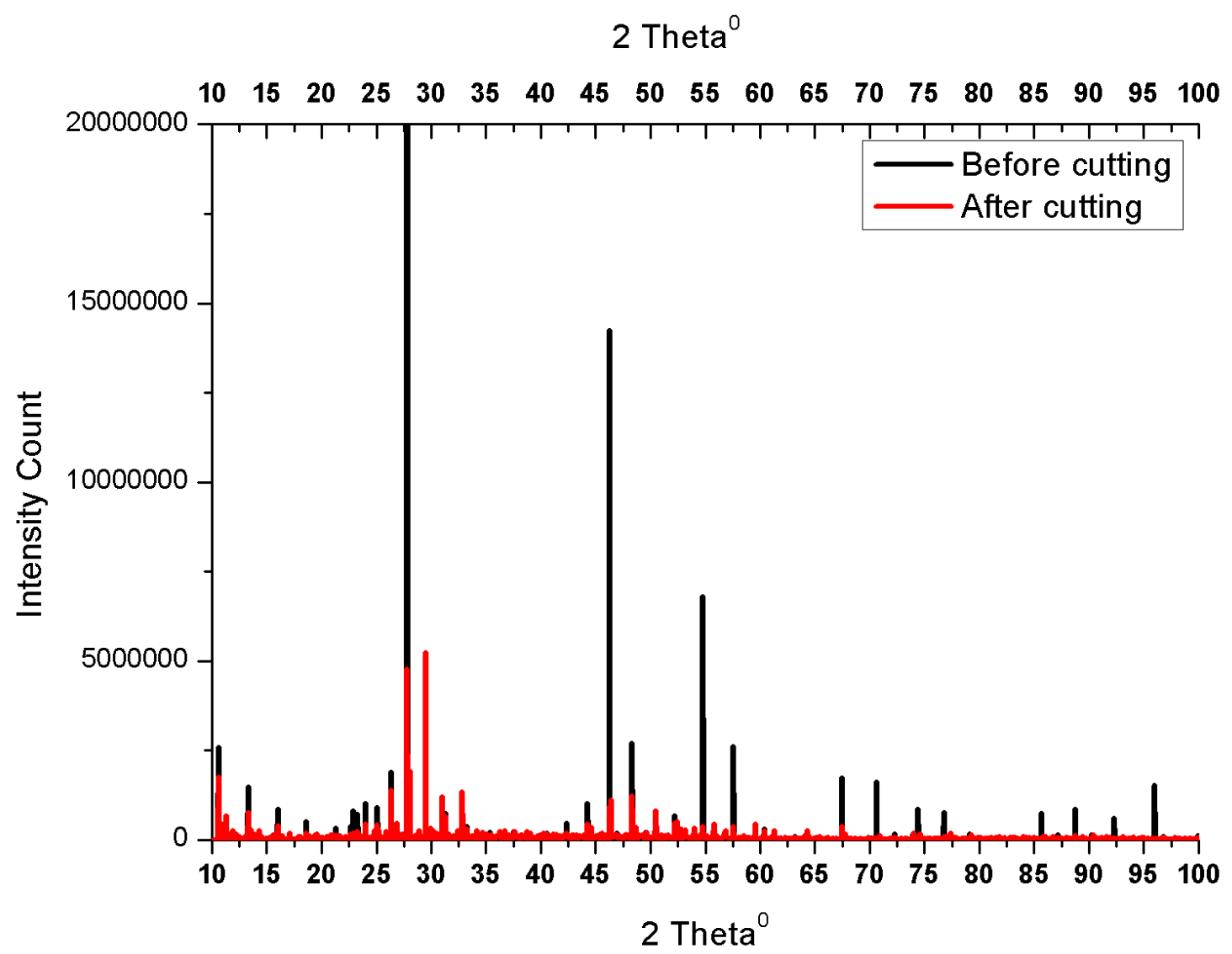

Figure 11: Simulated XRD spectra of silicon before and after cutting. Black peaks signifies crystalline silicon before cutting while scattered red peaks signifies solid state amorphisation of silicon after cutting. 

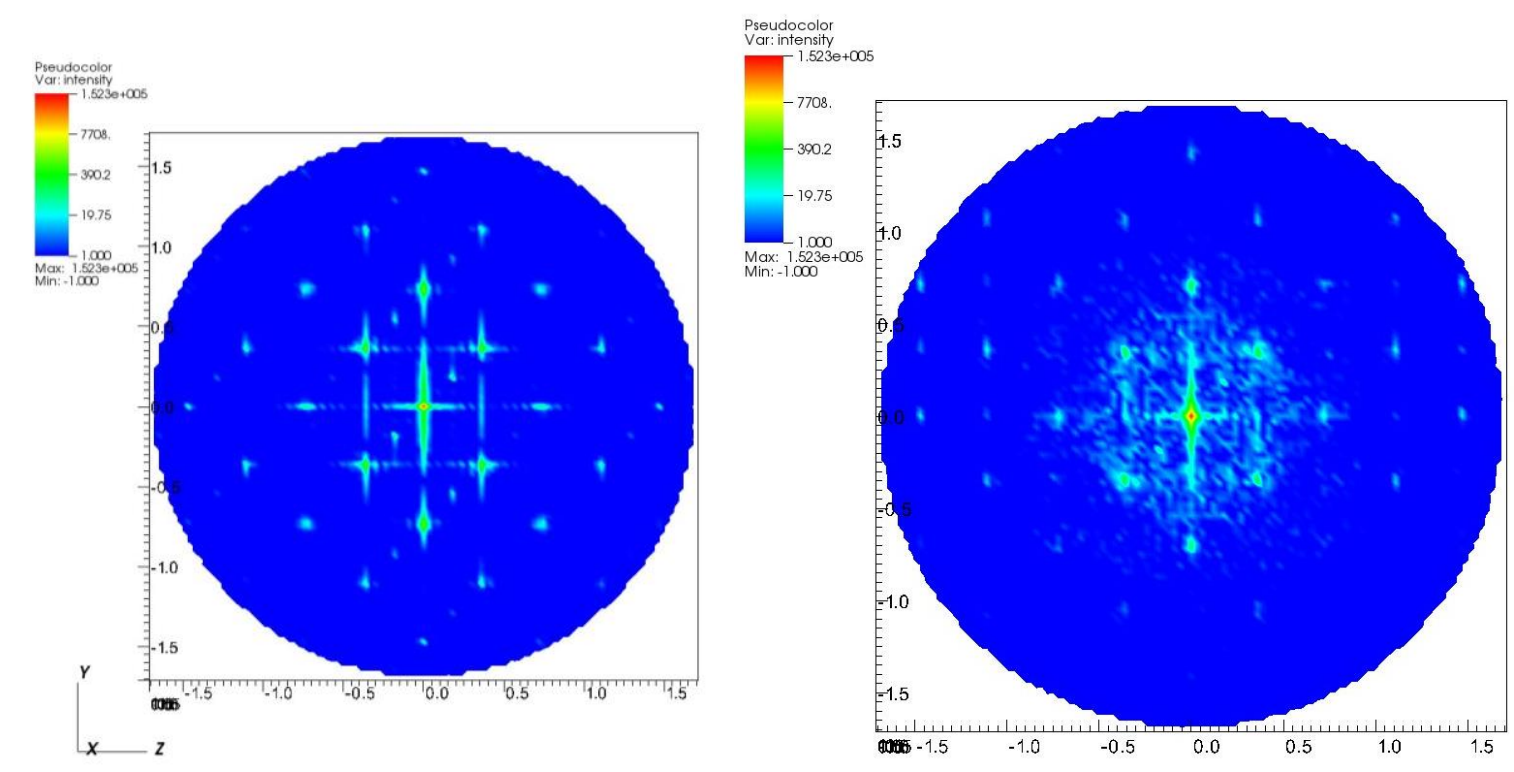

Figure 12: SAED patterns of silicon aligned with the (010) orientation (a) before cutting (b) after cutting. Change in the spot pattern before cutting diminishes after cutting. The colours in the pseudo colour bar shows the relative intensity of the diffraction patters calculated from the simulations.

\subsection{Presence of dislocations}

Figure 13a shows the simulation results which were obtained by post-processing of the MD trajectories using the dislocation extraction algorithm (DXA). It was anticipated that dislocation nucleation might occur during the process of nanometric cutting [41], but no dislocations were found to travel ahead of the tool that will drive plasticity in silicon (this is possible due to scale limitations of the MD) since experimental studies reported presence of several type of dislocations in contrast to what is observed here $[51,52]$. Careful examination of the simulation video showed some $1 / 4<111>$ partial dislocations in the sub-surface and not in the cutting zone. It may however be noted that nucleation of dislocations is somewhat a stochastic process and may not necessarily be guaranteed for such small cutting depths. For instance, as shown in figure 13, while the cutting was carried out with a polycrystalline diamond tool, some partial dislocations with Burgers vector $1 / 4<111>$ and perfect dislocations with Burgers vector $1 / 2<110>$ in the substrate's machined sub-surface were detected and these are summarised in table IV for a ready glance. Aside from these observations, the atoms of silicon can noticeably be seen to become amorphous, forming the cutting chips ahead of the cutting tool. The degree of amorphisation in the three simulation test cases was found to be different 
Paper accepted in the Journal "Acta Materialia" in November 2015

e.g. cutting chips of single crystal silicon were completely amorphous whereas the cutting chips of the polycrystalline substrate were found to contain small nano-crystallites, which did not undergo complete amorphisation

Table IV: Burgers Vector of the dislocation observed during the simulation

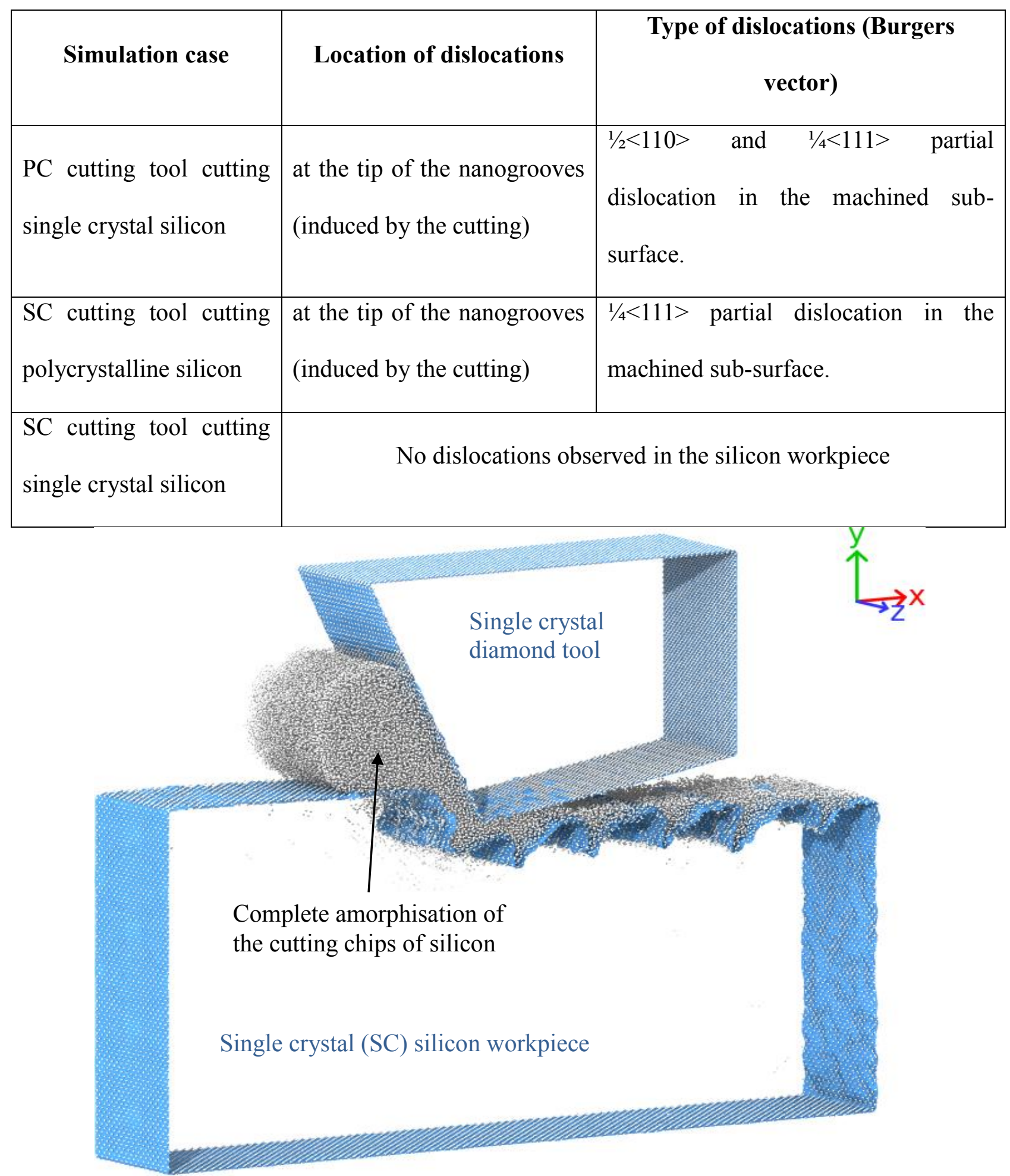

(a) Cutting of a single crystal workpiece with a single crystal cutting tool 
Paper accepted in the Journal "Acta Materialia" in November 2015

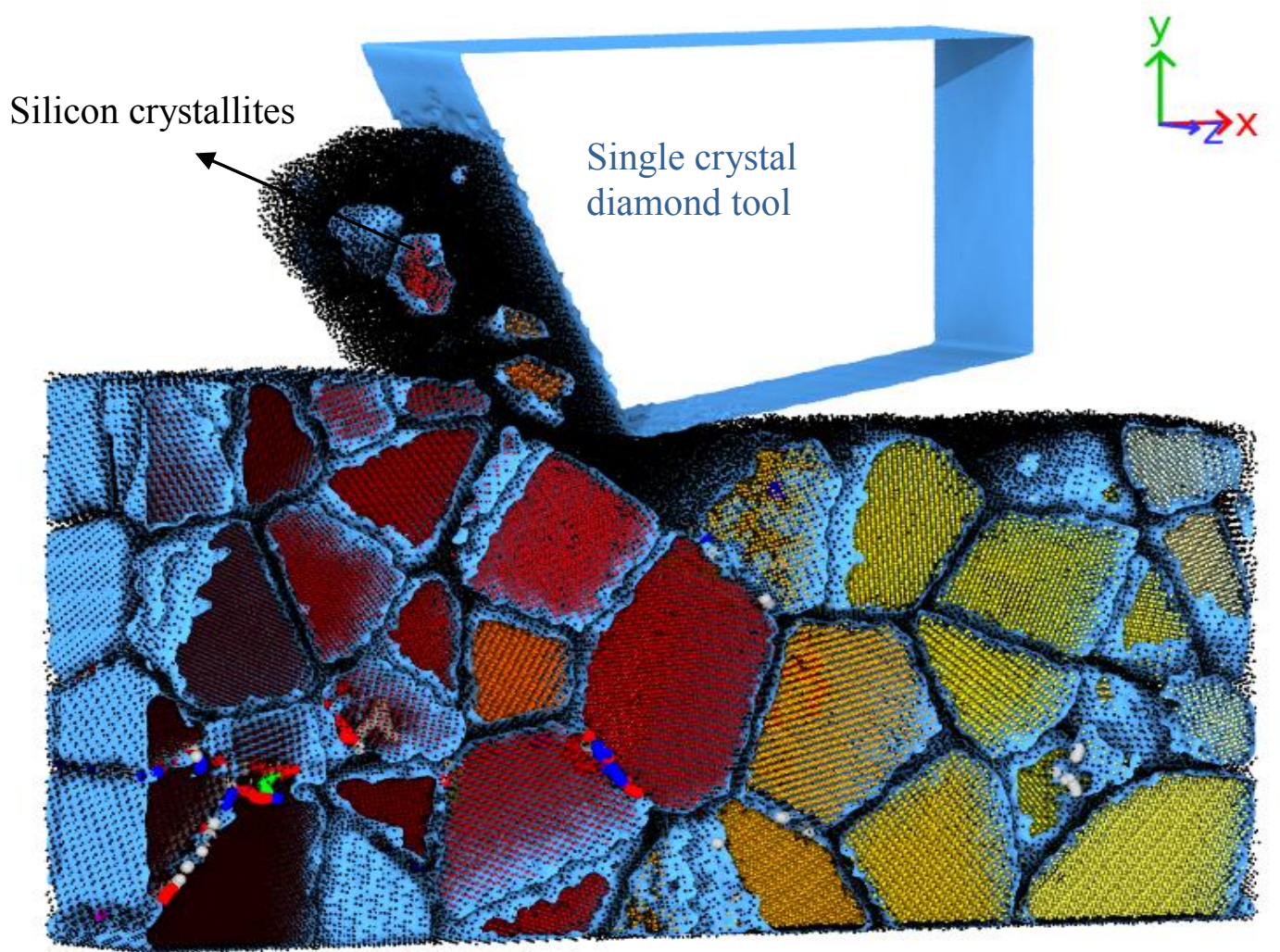

(b) Cutting of a polycrystalline workpiece with a single-crystal cutting tool

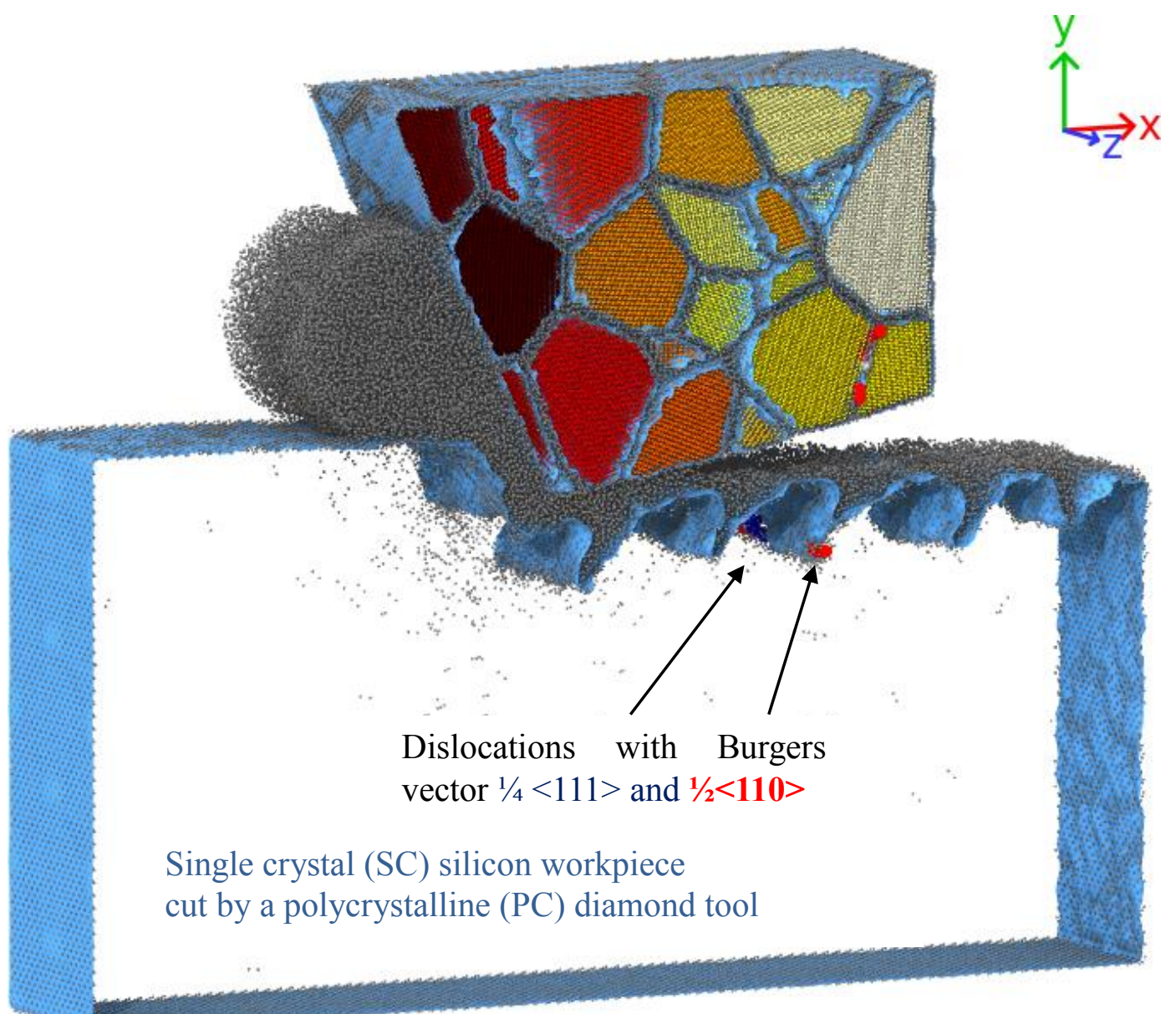

(c) Cutting of a single crystal workpiece with a polycrystalline cutting tool 
Paper accepted in the Journal "Acta Materialia" in November 2015

Figure 13: Output of the DXA algorithm showing snapshots of the nanometric cutting of silicon. The geometric boundaries of the workpiece and cutting tool and the respective grains are shown, while the geometric boundaries of the disordered phases are not visible in these visualizations. Colour of the atoms represent atomic cluster to which they belong.

\subsection{Pressure-Temperature variation}

In this MD simulation study, we propose to use the Minor Principal stress (see Appendix-1) as the main criterion to predict yielding of silicon during its nanometric cutting, motivated by the fact that, unlike von Mises stress, it can distinguish tension and compression. Figure 14 shows the average variation in the Minor Principal stress and temperature of a group of silicon atoms over time and cutting distance. Thus, it can be seen from figure 14 that peak temperature in the cutting zone went up to $1378 \mathrm{~K}$ and the Minor Principal stress approached $10 \mathrm{GPa}$. An interesting observation from figure 14 is that these two peak events did not coincide.

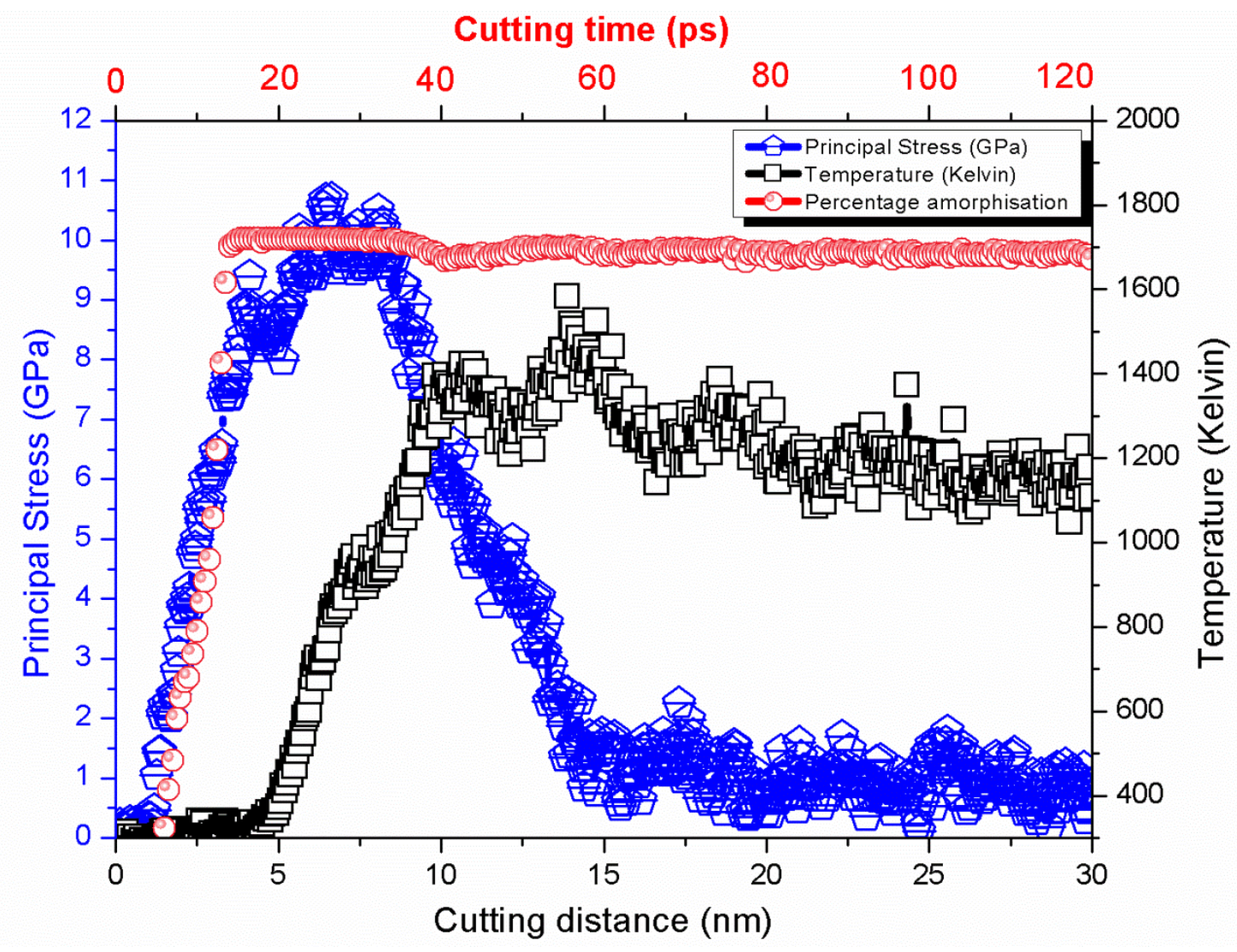

Figure 14: Variation in the Minor Principal stress, temperature and corresponding degree of amorphisation in the cutting zone of silicon. The amorphisation reaches $100 \%$ along with the sharp rise in Minor Principal stress.

Furthermore, the temperature peak lags the stress peak in the cutting zone. It was difficult to assert 
Paper accepted in the Journal "Acta Materialia" in November 2015

from this information as to whether the stress peak or the temperature peak causes amorphisation of silicon. In order to address this question, additional information was extracted using OVITO. The atoms in the monitoring group (shown earlier in figure 2) for measuring local stress and temperature were also used to quantify the transition from crystalline to amorphous state over time (red line in figure 14). Noticeably, the trend of amorphisation follows stress rather than temperature and $100 \%$ amorphisation was achieved typically at a stress of $10 \mathrm{GPa}$ while the temperature was still at $300 \mathrm{~K}$. This shows that nanometric cutting and hence the structural transformations observed during the ductile-regime cutting of silicon in MD simulations are an outcome of deviatoric stress in the cutting zone rather than temperature.

\section{Conclusions}

Despite a rich body of literature there are several gaps in the current pool of knowledge of highpressure surface science of silicon. An important question is whether differences exist in the plasticity during cutting of a single crystal silicon and polycrystalline silicon. Furthermore, from a modelling point of view, can the newly proposed screening functions address the gaps between the experiments and the previously published simulations? This is supplemented by the fact that no direct and conclusive evidence of the Si-I to Si-II transformation or high pressure phase transformation (HPPT) of silicon has been provided by previously published MD simulation studies, yet HPPT has constantly been reported as being the mechanism underlying the plastic response of silicon while it undergoes brittle-ductile transition. In view of these open questions, MD simulation was used in this work to study the process of nanometric cutting of polycrystalline and single crystalline silicon substrates and polycrystalline and single crystalline diamond cutting tools on the basis of a new long-ranged analytical bond order potential (screening functions). The following key conclusions can be drawn from the observations reported and discussed in this paper:

1. Direct amorphisation from the pristine crystalline phase, in contrast to HPPT, is identified as the root cause of plasticity in silicon in MD simulations. This contradicts the established 
Paper accepted in the Journal "Acta Materialia" in November 2015

experimental understanding that $\mathrm{Si}-\mathrm{I}$ phase first transforms to the $\beta$-Sn phase (Si-II) typically at a pressure of about $12 \mathrm{GPa}$. This discrepancy can be explained with the presence of crystal defects in real world materials while in perfect model crystals an activation barrier delays the HPPT.

2. The kinetics of the brittle-ductile transition (via amorphisation) preferentially follows the path of stress rather than the temperature i.e. amorphisation is a consequence of stress rather than the temperature. Thus, plasticity in silicon is triggered by large deviatoric stresses rather than the high temperature in the cutting zone. Typically, a Minor Principal stress of about $10 \mathrm{GPa}$ was observed to cause direct amorphisation in single crystal silicon. Interestingly, in polycrystalline silicon the magnitude of stress and thereby the degree of amorphisation were lower in comparison to single crystal silicon specimen. Also, the cutting of polycrystal silicon consumed least specific cutting energy (work done by the cutting tool in removing a unit volume of material) while cutting performed with a polycrystalline diamond-cutting tool consumed most specific cutting energy.

3. Only few dislocations were observed in the machined sub-surface of silicon. However, corroborating with experiments, the formation of periodic nanogrooves oriented at an angle of $45^{\circ}$ to $55^{\circ}$ in the sub-surface of silicon was observed, a unique phenomenon that has not been obtainable using classical (short-ranged) Tersoff or analytical bond order potential functions. The nanogrooves were recognized as sites of brittle fracture which grow further and penetrate deeper into the sub-surface of the cut silicon substrate due to the pressing flowing amorphous phase of silicon. This phenomenon explains that, while negative inclination of the probe or the cutting tool facilitates a ductile response from brittle materials, it also results in increasing sub-surface damage to the substrate.

\section{Acknowledgments:}

SG would like to acknowledge the financial support in the form of 'Short Term Scientific Mission' from the COST Action MP1303 (STSM MP1303-30208) of the Horizon 2020 program as well as 
Paper accepted in the Journal "Acta Materialia" in November 2015

addition support from the planning grant from Invest NI (LoO: 1504/101356075) and International

Research Fellowship account of Queen's University, Belfast in undertaking this work. GC acknowledges support from the Science Foundation of Ireland (08/IN.1/I1932). The authors would also like to acknowledge the use of HPC service of STFC Hartree Centre.

\section{References:}

[1] S. Goel, X. Luo, A. Agrawal, R.L. Reuben. Diamond machining of silicon: A review of advances in molecular dynamics simulation, International Journal of Machine Tools and Manufacture 88 (2015) 131-164.

[2] K. Mylvaganam, L.C. Zhang. Nanotwinning in monocrystalline silicon upon nanoscratching, Scripta Mater 65 (2011) 214-216.

[3] A.M. Minor §, E.T. Lilleodden, M. Jin, E.A. Stach, D.C. Chrzan, J.W. Morris. Room temperature dislocation plasticity in silicon, Philos Mag 85 (2005) 323-330.

[4] G.L.W. Cross. Silicon nanoparticles: Isolation leads to change, Nat Nano 6 (2011) 467-468.

[5] D. Chrobak, N. Tymiak, A. Beaber, O. Ugurlu, W.W. Gerberich, R. Nowak. Deconfinement leads to changes in the nanoscale plasticity of silicon, Nat Nano 6 (2011) 480-484.

[6] J.B. Pethicai, R. Hutchings, W.C. Oliver. Hardness measurement at penetration depths as small as $20 \mathrm{~nm}$, Philosophical Magazine A 48 (1983) 593-606.

[7] $\mathrm{H}$. Wu. PhD Thesis on Fundamental investigations of cutting of silicon for photovoltaic applications. George W. Woodruff School of Mechanical Engineering, vol. PhD. Georgia, USA: Georgia Institute of Technology, 2012.

[8] M. Brede. The brittle-to-ductile transition in silicon, Acta Metall Mater 41 (1993) 211-228.

[9] M. Brede, P. Haasen. The brittle-to-ductile transition in doped silicon as a model substance, Acta Metallurgica 36 (1988) 2003-2018.

[10] R.W. Cahn. Metallic solid silicon, Nature 357 (1992) 645-646.

[11] Q.H. Fang, L.C. Zhang. Prediction of the threshold load of dislocation emission in silicon during nanoscratching, Acta Mater 61 (2013) 5469-5476.

[12] J. Tersoff. Modeling solid-state chemistry: Interatomic potentials for multicomponent systems, Phys Rev B 39 (1989) 5566.

[13] J. Tersoff. Erratum: Modeling solid-state chemistry: Interatomic potentials for multicomponent systems, Phys Rev B 41 (1990) 3248.

[14] P. Erhart, K. Albe. Analytical potential for atomistic simulations of silicon, carbon, and silicon carbide, Phys Rev B 71 (2005) 035211.

[15] L. Pastewka, A. Klemenz, P. Gumbsch, M. Moseler. Screened empirical bond-order potentials for Si-C, Phys Rev B 87 (2013) 205410.

[16] W.C.D. Cheong, L.C. Zhang. Molecular dynamics simulation of phase transformations in silicon monocrystals due to nano-indentation, Nanotechnology 11 (2000) 173.

[17] C. Sanz-Navarro, S. Kenny, R. Smith. Atomistic simulations of structural transformations of silicon surfaces under nanoindentation, Nanotechnology 15 (2004) 692.

[18] D. Kim, S. Oh. Atomistic simulation of structural phase transformations in monocrystalline silicon induced by nanoindentation, Nanotechnology 17 (2006) 2259.

[19] K. Mizushima, S. Yip, E. Kaxiras. Ideal crystal stability and pressure-induced phase transition in silicon, Physical Review B 50 (1994) 14952.

[20] S. Goel, X. Luo, P. Comley, R.L. Reuben, A. Cox. Brittle-ductile transition during diamond turning of single crystal silicon carbide, International Journal of Machine Tools and Manufacture 65 (2013) 15-21.

[21] O. Auciello, A.V. Sumant. Status review of the science and technology of ultrananocrystalline diamond (UNCD ${ }^{\mathrm{TM}}$ ) films and application to multifunctional devices, Diam Relat Mater 19 (2010) 
699-718.

[22] S. Plimpton. Fast Parallel Algorithms for Short-Range Molecular Dynamics, J Comput Phys 117 (1995) 1-19.

[23] A. Stukowski. Visualization and analysis of atomistic simulation data with OVITO-the Open Visualization Tool, Model Simul Mater Sc 18 (2010).

[24] A. Stukowski, V.V. Bulatov, A. Arsenlis. Automated identification and indexing of dislocations in crystal interfaces, Model Simul Mater Sc 20 (2012) 085007.

[25] A. Stukowski, K. Albe. Extracting dislocations and non-dislocation crystal defects from atomistic simulation data, Model Simul Mater Sc 18 (2010) 085001.

[26] S. Goel, X. Luo, R.L. Reuben, W.B. Rashid. Replacing diamond cutting tools with CBN for efficient nanometric cutting of silicon, Mater Lett 68 (2012) 507-509.

[27] S. Goel, N.H. Faisal, V. Ratia, A. Agrawal, A. Stukowski. Atomistic investigation on the structure-property relationship during thermal spray nanoparticle impact, Comp Mater Sci 84 (2014) 163-174.

[28] S. Goel, X. Luo, R. Reuben, W. Rashid. Atomistic aspects of ductile responses of cubic silicon carbide during nanometric cutting, Nanoscale Research Letters 6 (2011) 589.

[29] S. Goel, X. Luo, R.L. Reuben. Shear instability of nanocrystalline silicon carbide during nanometric cutting, Appl Phys Lett 100 (2012) 231902.

[30] S. Goel, A. Stukowski, X. Luo, A. Agrawal, R.L. Reuben. Anisotropy of single-crystal 3CSiC during nanometric cutting, Model Simul Mater Sc 21 (2013) 065004.

[31] V.I. Yamakov, D.H. Warner, R.J. Zamora, E. Saether, W.A. Curtin, E.H. Glaessgen. Investigation of crack tip dislocation emission in aluminum using multiscale molecular dynamics simulation and continuum modeling, J Mech Phys Solids 65 (2014) 35-53.

[32] Y. Jiwang, Z. Hongwei, K. Tsunemoto. Effects of tool edge radius on ductile machining of silicon: an investigation by FEM, Semicond Sci Tech 24 (2009) 075018.

[33] J. Yan, Syoji, Katsuo, Kuriyagawa, Tsunemoto, Suzuki, Hirofumi. Ductile regime turning at large tool feed, J Mater Process Tech 121 (2002) 363-372.

[34] S. Goel, N.H. Faisal, X. Luo, J. Yan, A. Agrawal. Nanoindentation of polysilicon and single crystal silicon: Molecular dynamics simulation and experimental validation, Journal of Physics D: Applied Physics 47 (2014) 275304.

[35] J. Schäfer, A. Stukowski, K. Albe. Plastic deformation of nanocrystalline Pd-Au alloys: On the interplay of grain boundary solute segregation, fault energies and grain size, Acta Mater 59 (2011) 2957-2968.

[36] P.M. Agrawal, L.M. Raff, R. Komanduri. Monte Carlo simulations of void-nucleated melting of silicon via modification in the Tersoff potential parameters, Phys Rev B 72 (2005) 125206.

[37] Y.A. Du, T.J. Lenosky, R.G. Hennig, S. Goedecker, J.W. Wilkins. Energy landscape of silicon tetra-interstitials using an optimized classical potential, physica status solidi (b) 248 (2011) 20502055.

[38] F. Shimizu, S. Ogata, J. Li. Theory of shear banding in metallic glasses and molecular dynamics calculations, Mater Trans 48 (2007) 2923-2927.

[39] A. Heidelberg, L.T. Ngo, B. Wu, M.A. Phillips, S. Sharma, T.I. Kamins, J.E. Sader, J.J. Boland. A generalized description of the elastic properties of nanowires, Nano Lett 6 (2006) 11011106.

[40] G.E. Dieter, D. Bacon. Mechanical metallurgy, McGraw-Hill New York, 1986.

[41] K. Puttick, L. Whitmore, C. Chao, A. Gee. Transmission electron microscopy of nanomachined silicon crystals, Philosophical Magazine A 69 (1994) 91-103.

[42] C. Saeed Zare, G. Saurav, L. Xichun. Molecular dynamics simulation investigation on the plastic flow behaviour of silicon during nanometric cutting, Model Simul Mater Sc 24 (2016) 015002. [43] G.L. Cross, B.S. O'Connell, H.O. Özer, J.B. Pethica. Room temperature mechanical thinning and imprinting of solid films, Nano Lett 7 (2007) 357-362.

[44] M. Lai, X.D. Zhang, F.Z. Fang. Study on critical rake angle in nanometric cutting, Appl. Phys. A 108 (2012) 809-818. 
Paper accepted in the Journal "Acta Materialia" in November 2015

[45] S. Goel, X. Luo, R.L. Reuben. Wear mechanism of diamond tools against single crystal silicon in single point diamond turning process, Tribol Int 57 (2013) 272-281.

[46] S. Goel, X. Luo, R.L. Reuben, H. Pen. Influence of temperature and crystal orientation on tool wear during single point diamond turning of silicon, Wear 284-285 (2012) 65-72.

[47] J.J. Gilman. Mechanism of shear-induced metallization, Czech J Phys 45 (1995) 913-919.

[48] S.P. Coleman, D.E. Spearot, L. Capolungo. Virtual diffraction analysis of Ni $\left[\begin{array}{lll}0 & 1 & 0\end{array}\right]$ symmetric tilt grain boundaries, Model Simul Mater Sc 21 (2013) 055020.

[49] S. Patra, P. Mitra, S.K. Pradhan. Preparation of nanodimensional CdS by chemical dipping technique and their characterization, Materials Research 14 (2011) 17-20.

[50] K. Pandey, N. Garg, K. Shanavas, S.M. Sharma, S. Sikka. Pressure induced crystallization in amorphous silicon, J Appl Phys 109 (2011) 113511.

[51] T. Shibata, S. Fujii, E. Makino, M. Ikeda. Ductile-regime turning mechanism of single-crystal silicon, Precision Engineering 18 (1996) 129-137.

[52] J. Yan, T. Asami, H. Harada, T. Kuriyagawa. Fundamental investigation of subsurface damage in single crystalline silicon caused by diamond machining, Precision Engineering 33 (2009) 378-386. 
Stress tensor $=\left[\begin{array}{lll}\sigma_{x x} & \tau_{x y} & \tau_{x z} \\ \tau_{x y} & \sigma_{y y} & \tau_{y z} \\ \tau_{x z} & \tau_{x z} & \sigma_{z z}\end{array}\right]$

Invariants:

$\mathrm{I}_{1}=\sigma_{\mathrm{xx}}+\sigma_{\mathrm{yy}}+\sigma_{\mathrm{zz}}$

$\mathrm{I}_{2}=\sigma_{\mathrm{xx}} \sigma_{\mathrm{yy}}+\sigma_{\mathrm{yy}} \sigma_{z z}+\sigma_{z z} \sigma_{x x}-\tau_{x y}{ }^{2}-\tau_{x z}{ }^{2}-\tau_{y z}{ }^{2}$

$\mathrm{I}_{3}=\sigma_{\mathrm{xx}} \sigma_{\mathrm{yy}} \sigma_{\mathrm{zz}}+2\left(\tau_{x y} \tau_{y z} \tau_{x z}\right)-\tau_{x z}{ }^{2} \sigma_{y y}-\tau_{y z}{ }^{2} \sigma_{x x}-\tau_{x y}{ }^{2} \sigma_{z z}$

$\mathrm{A}_{1}=-\mathrm{I}_{1} ; \quad A_{2}=I_{2} \quad A_{3}=-I_{3}$

$\mathrm{Q}=\frac{3 \mathrm{~A}_{2}-\mathrm{A}_{1}^{2}}{9}$

$\mathrm{R}=\frac{9 \mathrm{~A}_{1} \mathrm{~A}_{2}-27 \mathrm{~A}_{3}-2 A_{1}^{3}}{54}$

$\mathrm{D}=Q^{3}+R^{2}$

If $\mathrm{D}<0$ then as follows: else the condition is $2 \mathrm{D}$ stress

$$
\begin{aligned}
& \theta=\cos ^{-1}\left(\frac{R}{\sqrt{-Q^{3}}}\right) \\
& R_{1}=2 \sqrt{-Q} \times \cos \left(\frac{\theta}{3}\right)-\frac{A_{1}}{3} \\
& R_{2}=2 \sqrt{-Q} \times \cos \left(\frac{\theta+4 \pi}{3}\right)-\frac{A_{1}}{3} \\
& R_{3}=2 \sqrt{-Q} \times \cos \left(\frac{\theta+2 \pi}{3}\right)-\frac{A_{1}}{3}
\end{aligned}
$$

Major principalstress $\left(\sigma_{1}\right)=\max \left(R_{1}, R_{2}, R_{3}\right) \quad ; \operatorname{Minor} \operatorname{principalstress}\left(\sigma_{3}\right)=\min \left(R_{1}, R_{2}, R_{3}\right)$ 
Paper accepted in the Journal "Acta Materialia" in November 2015

$\sigma_{\text {tresca }}=\frac{\sigma_{1}-\sigma_{3}}{2}$

$\sigma_{\text {von Mises }}=\sqrt{\frac{\left(\sigma_{\mathrm{xx}}-\sigma_{\mathrm{yy}}\right)^{2}+\left(\sigma_{\mathrm{yy}}-\sigma_{\mathrm{zz}}\right)^{2}+\left(\sigma_{\mathrm{zz}}-\sigma_{\mathrm{xx}}\right)^{2}+6\left(\tau_{\mathrm{xy}}^{2}+\tau_{\mathrm{yz}}^{2}+\tau_{\mathrm{zx}}^{2}\right)}{2}}$

$\sigma_{\text {octahedral }}=\frac{\sqrt{\left(\sigma_{\mathrm{xx}}-\sigma_{\mathrm{yy}}\right)^{2}+\left(\sigma_{\mathrm{yy}}-\sigma_{\mathrm{zz}}\right)^{2}+\left(\sigma_{\mathrm{zz}}-\sigma_{\mathrm{xx}}\right)^{2}+6\left(\tau_{\mathrm{xy}}^{2}+\tau_{\mathrm{yz}}^{2}+\tau_{\mathrm{zx}}^{2}\right)}}{3}=\frac{\sqrt{2}}{3} \sigma_{\text {von Mises }}$ 